NATIONAL AERONAUTICS AND SPACE ADMINISTRATION

Technical Report No. 32-984

\title{
Ground Instrumentation for Mariner IV Occultation Experiment
}
G. S. Levy
T. Y. Otoshi
B. L. Seidel

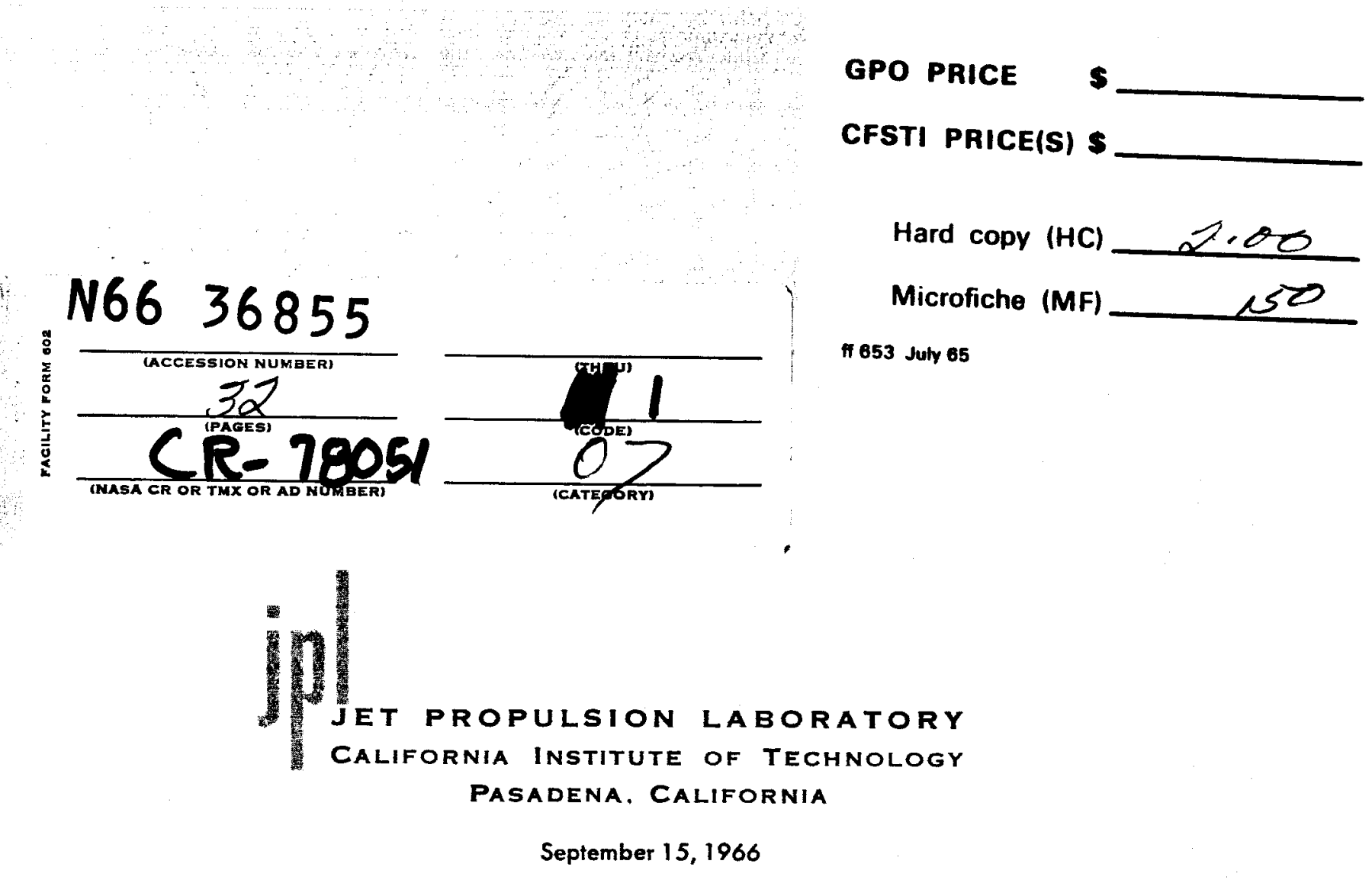


NATIONAL AERONAUTICS AND SPACE ADMINISTRATION

Technical Report No. 32-984

\title{
Ground Instrumentation for Mariner IV Occultation Experiment
}

\author{
G. S. Levy \\ T. Y. Otoshi \\ B. L. Seidel
}

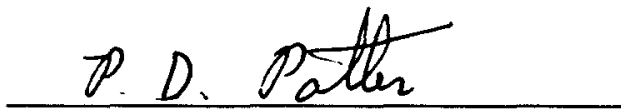

P. D. Potter, Manager

Communications Elements Research Section

\begin{abstract}
JET PROPULSION LABORATORY
CALIFORNIA INSTITUTE OF TECHNOLOGY

Pasadena. California
\end{abstract}


Copyright (C) 1966

Jef Propulsion Laboratory

California Institute of Technology

Prepared Under Contract No. NAS 7-100

National Aeronautics \& Space Administration 


\section{CONTENTS}

I. Introduction . . . . . . . . . . . . . . . . . . . . . 1

A. Description of Experiment . . . . . . . . . . . . . . . 1

B. Ground Instrumentation Requirements . . . . . . . . . . . 2

II. DSIF Radio System . . . . . . . . . . . . . . . . . . . . . . . . . . . 4

A. General Description. . . . . . . . . . . . . . . . . . 4

B. DSIF Phase-Lock-Loop Receiver . . . . . . . . . . . . . 4

C. Open-Loop Occultation Receiver . . . . . . . . . . . . . 5

D. Tape Recording Subsystem . . . . . . . . . . . . . . . . 6

III. Receiver and Spacecraft System Tests . . . . . . . . . . . . . 9

A. Phase-Lock-Loop Receiver Tests . . . . . . . . . . . . . . . 9

1. Doppler Extraction Tests . . . . . . . . . . . . . . . . . . . 9

2. Closed-Loop AGC Tests . . . . . . . . . . . . . . . . . . 9

a. Frequency Response . . . . . . . . . . . . . . . 9

b. Dynamic AGC Calibrations . . . . . . . . . . . . . 11

c. Static AGC Calibrations . . . . . . . . . . . . . . 13

B. Open-Loop Occultation Receiver Tests . . . . . . . . . . . 14

C. Spacecraft Transponder Acquisition-Time Tests . . . . . . . . . 14

D. Simulation of Expected-Amplitude Modulation . . . . . . . . . 16

IV. Results of the Mariner IV Occultation Experiment . . . . . . . 19

References . . . . . . . . . . . . . . . . . . 22

Table 1. FR 1200/100 tape recorder track assginments $\quad$. . . . . . 8

\section{FIGURES}

1. Mariner IV occultation sequence . . . . . . . . . . . . . . . 2

2. Amplitude modulation due to Fresnel diffraction effect . . . . . . . 3

3. Radio system for Mariner IV Occultation Experiment . . . . . . . . . 4

4. DSIF phase-lock-loop receiver system . . . . . . . . . . . . . 5

5. Open-loop occultation receiver instrumentation . . . . . . . . . . 6

6. DSIF tape recording subsystem . . . . . . . . . . . . . . . . 6 


\section{FIGURES (Cont'd)}

7. Occultation experiment tape recording configuration . . . . . . . . 7

8. One-second cumulative doppler standard deviation . . . . . . . . . 9

9. Equivalent AGC loop for dynamic signal conditions (noise-free case) . . . 10

10. Dynamic AGC frequency response . . . . . . . . . . . . . . . . . 11

11. Static AGC frequency response . . . . . . . . . . . . . . . 12

12. Normalized RF signal voltage vs dynamic AGC voltage . . . . . . . 13

13. AGC characteristic curve . . . . . . . . . . . . . . . . . . 13

14. Power spectral density of Mariner $N$ signal using 24.6-eps window . . . 14

15. Power spectral density of Mariner IV signal using 6.2-cps window . . 15

16. Transponder lock-on times vs frequency offset . . . . . . . . . . . . 16

17. Number of 10-sec counts vs VCO frequency when not in lock . . . . . 16

18. Occulfation simulation, input power $-120 \mathrm{dbm}$, receiver 1 in MGC mode . . . . . . . . . . . . . . . . . . . . . 17

19. Occultation simulation, input power $-120 \mathrm{dbm}$, receiver 1 in AGC mode . . . . . . . . . . . . . . . . . . . . . 17

20. Occultation simulation, input power $-158 \mathrm{dbm}$, receiver 1 in MGC mode . . . . . . . . . . . . . . . . . . . . . 18

21. Occulfation simulation, input power $-158 \mathrm{dbm}$, receiver 1

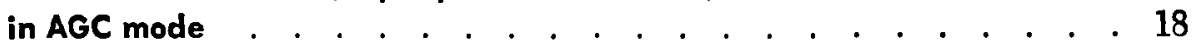

22. Entrance occultation closed-loop doppler residuals . . . . . . . . . . 19

23. Entry residual sum, closed loop . . . . . . . . . . . . . . . . . . 19

24. Wide-band open-loop spectrum 3:25:17, DSIF-12 site . . . . . . . . . 20

25. Narrow-band open-loop spectrum 3:25:17, DSIF-12 site . . . . . . . . 20

26. Entrance occultation doppler residuals, DSIF-12 open- and closed-loop receivers . . . . . . . . . . . . . . . . 21

27. Broad-band spectrum of transition from auxiliary oscillator to VCO . . . 21

28. Doppler residuals from exit of occultation from DSIF-11 and -12 open-loop receivers 


\begin{abstract}
The Mariner IV Occultation Experiment imposed unique requirements upon the NASA/Jet Propulsion Laboratory Deep Space Instrumentation Facility. It was necessary to measure extremely small deviations in the rate of change of frequency to a few parts in $10^{10}$. The instrumentation used and the results obtained are described.
\end{abstract}

\title{
I. INTRODUCTION
}

\section{A. Description of Experiment}

The determination of the Martian atmospheric parameters was the objective of the Mariner IV Occultation Experiment. This was accomplished by the measurement of doppler frequency perturbations to the S-band telemetry link produced by the refractive index profile of the planetary atmosphere (Ref. 1).

After traveling over $71 / 2$ months in space, the Mariner IV spacecraft reached its closest point of approach to the planet Mars on July 15, 1965, at 01:00:58 GMT. At that time, Mariner $I V$ was approximately 6,100 miles from Mars and 134 million miles from Earth. The signal strength being received by Earth-based Deep Space Instrumentation Facility (DSIF) tracking stations was approximately $-157 \mathrm{dbm}$.

Approximately one hour after its closest approach to Mars, the Mariner IV spacecraft was occulted by the limb of the planet and remained in occultation for about $50 \mathrm{~min}$ (see Fig. 1). Two periods for obtaining data oc- curred: first, as the spacecraft was occulted by Mars; and, again, as it emerged from the opposite limb. Immediately before and after occultation, the spacecraft's $2300-\mathrm{Mc}$ tracking and telemetry signal traversed the atmosphere and ionosphere of Mars. The changes caused in the frequency, phase, and amplitude of the spacecraft's signal by passage through these media constituted the raw data of the experiment.

The Earth-based receiving stations involved in the occultation experiment may be seen in Fig. 1. At Goldstone, California, DSIF-11 (Pioneer) and DSIF-12 (Echo) each employed one standard DSIF phase-lock-loop receiver and one triple-conversion open-loop receiver. Near Canberra, Australia, DSIF-42 (Tidbinbilla) employed two DSIF phase-lock-loop receivers. These two types of receiver systems are discussed in Section II. The highpower transmitter at DSIF-13 (Venus), at Goldstone, was used to transmit $100-\mathrm{kw}$ signals to the spacecraft in an attempt to lock up the spacecraft transponder so that two-way doppler could be obtained the instant the spacecraft emerged from occultation. 


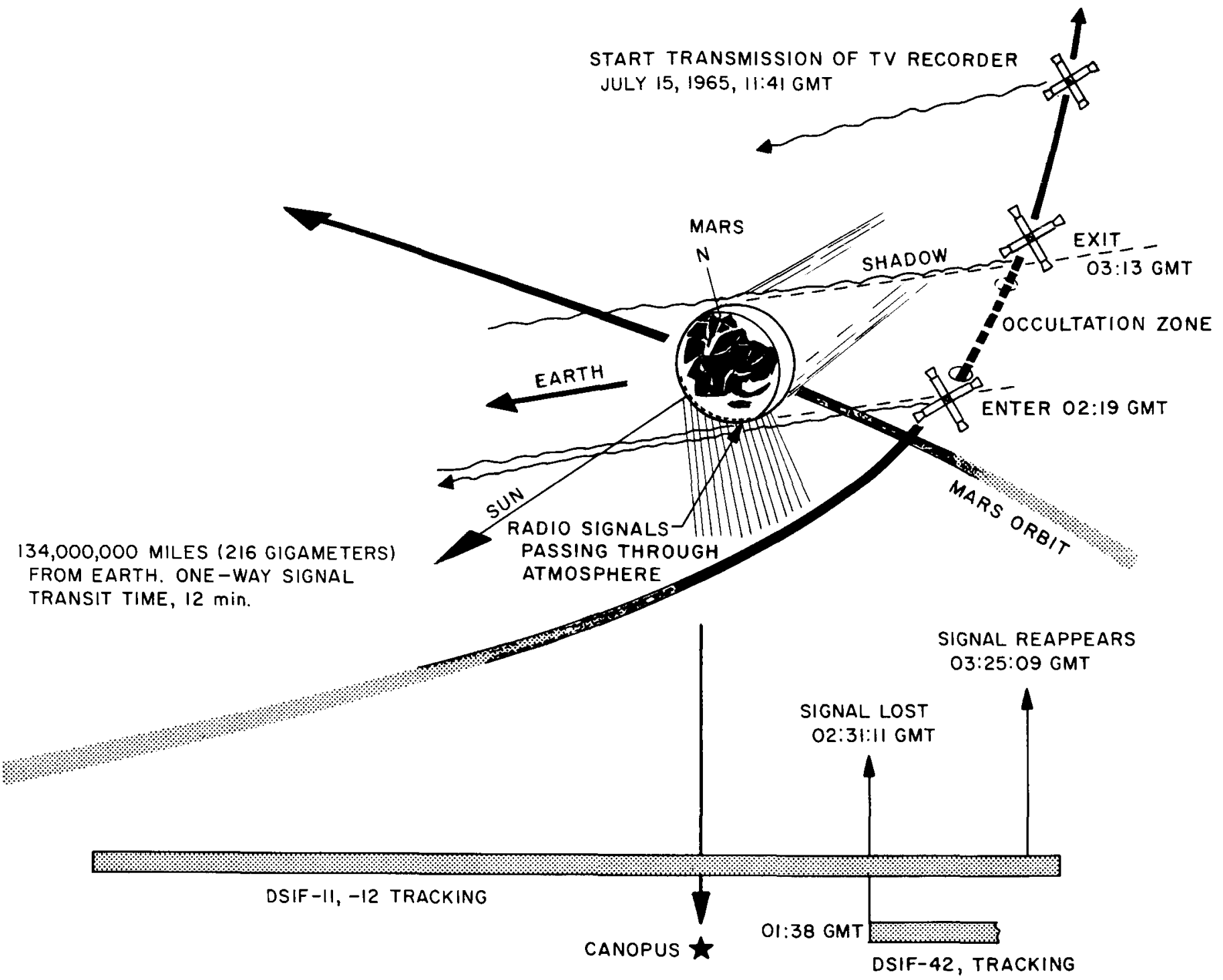

Fig. 1. Mariner IV occultation sequence

\section{B. Ground Instrumentation Requirements}

The principal quantities measured at the DSIF receiver stations during the occultation experiment were doppler frequency, $\mathrm{RF}$ phase, and signal strength as a function of time.

The Mariner IV Occultation Experiment placed unique demands on the measurement capabilities of the DSIF. The receiver equipment was required to have the accuracy and stability for measuring small perturbations of the doppler frequency, phase, and received-signal level as the spacecraft signals traversed the Martian ionosphere and atmosphere. The perturbation to the frequency was only a few parts in $10^{9}$. At the time of en- counter, the nominal received-signal strength was $-157 \mathrm{dbm}$, or approximately $16 \mathrm{db}$ above the threshold of the carrier loop of the standard DSIF phase-lock-loop receiver. It was expected that the Martian atmosphere might cause refractive attenuation (Refs. 1 and 2), hence change the amplitude of the signal received from the spacecraft. In addition, fluctuations in amplitude were predicted due to Fresnel diffraction at the planetary limb (Ref. 3). The atmosphere perturbs the diffraction pattern by tending to stretch it out, so that the period of the amplitude oscillations is increased. This effect, called the Fresnel stretch ( $F$ in Fig. 2), is also a measure of the atmospheric refractive index at the surface of the planet. 
The occultation measurements differed from those ordinarily made by the DSIF in tracking missions in that the time duration of the phenomena was short. The duration of measurable perturbations upon the frequency of the signals passing through the Martian troposphere and ionosphere was predicted to be between $20 \mathrm{sec}$ and $2 \mathrm{~min}$ (Ref. 1). Because the important portions of amplitude fluctuations due to Fresnel diffraction were expected to occur over a 1- to 10-sec period, the integration techniques normally used to obtain precise data could not be applied in this experiment.

In order to utilize the short-duration occultation signal information to fullest advantage, the decision was made to magnetically tape record specific parameters of the received signal during the occultation period. The information on the magnetic tapes could later be digitized, and processed by the use of various data reduction techniques. Parameters of the received signal which were recorded during occultation are described in Section II.D.

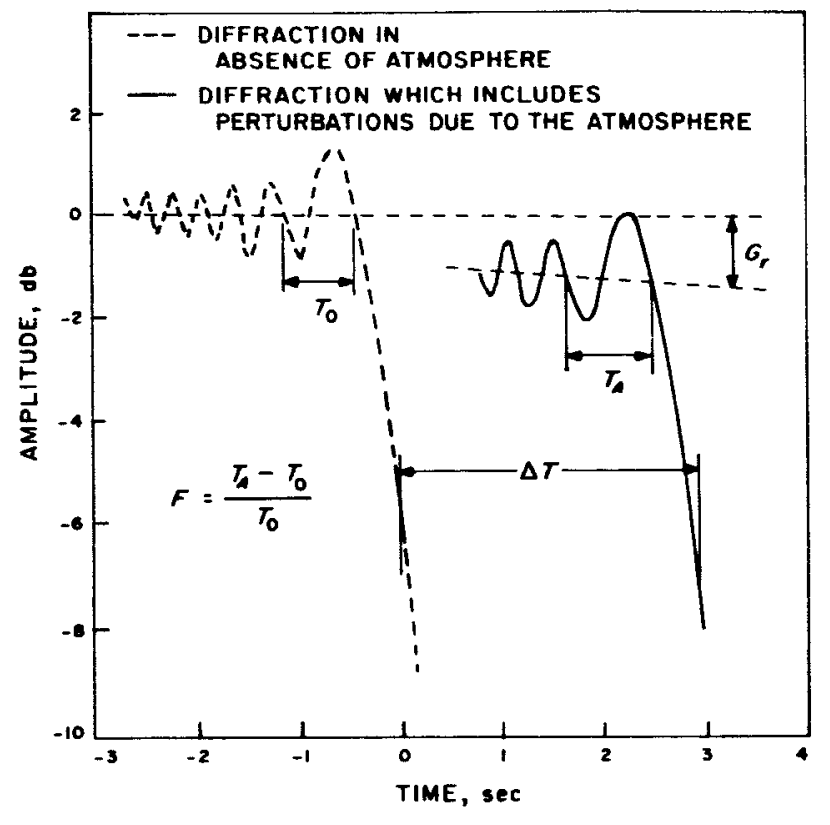

Fig. 2. Amplitude modulation due to Fresnel diffraction effect 


\section{DSIF RADIO SYSTEM}

\section{A. General Description}

The block diagram in Fig. 3 depicts the complete radio system employed. The ground station uses a rubidium standard to dirive a frequency synthesizer, which produces a signal of approximately $22 \mathrm{Mc}$. This signal is then modulated, multiplied 96 times in frequency, amplified, and transmitted to the spacecraft. The spacecraft radio system coherently translates the frequency and phase of the approximate $2.11-G c$ received signal by the ratio of 240 to 221 , thus yielding a carrier frequency of approximately $2.297 \mathrm{Gc}$. When the spacecraft receiver is in lock with the ground-station signal, the $2.297-\mathrm{Gc}$ signal is derived from the receiver's voltagecontrolled oscillator (VCO), which is phaselocked to the received signal. When no uplink signal is received, however, the RF signal is derived from a free-running crystal oscillator in the spacecraft. The RF signal is amplified and transmitted from a high-gain spacecraft antenna.
Each ground transmitter and receiver system employs an 85 -ft parabolic antenna with a Cassegrainian simultaneous-lobing feed. The receiver's front end employs a traveling-wave maser cooled by a closed-cycle helium refrigerator operating at about $4.2^{\circ} \mathrm{K}$. After amplification by the maser, the signal is split into phaselock-loop and open-loop receiver channels.

\section{B. DSIF Phase-Lock-Loop Receiver}

Figure 4 is a photograph of a DSIF S-band receiver system. It is basically a triple-conversion phaselocked receiver which yields power and frequency information in real time.

The receiver's VCO is kept in phase synchronism with the received signal. By a series of frequency multiplications, divisions, and additions, the ground-transmitter exciter frequency is coherently compared with the receiver VCO to obtain the two-way doppler frequency
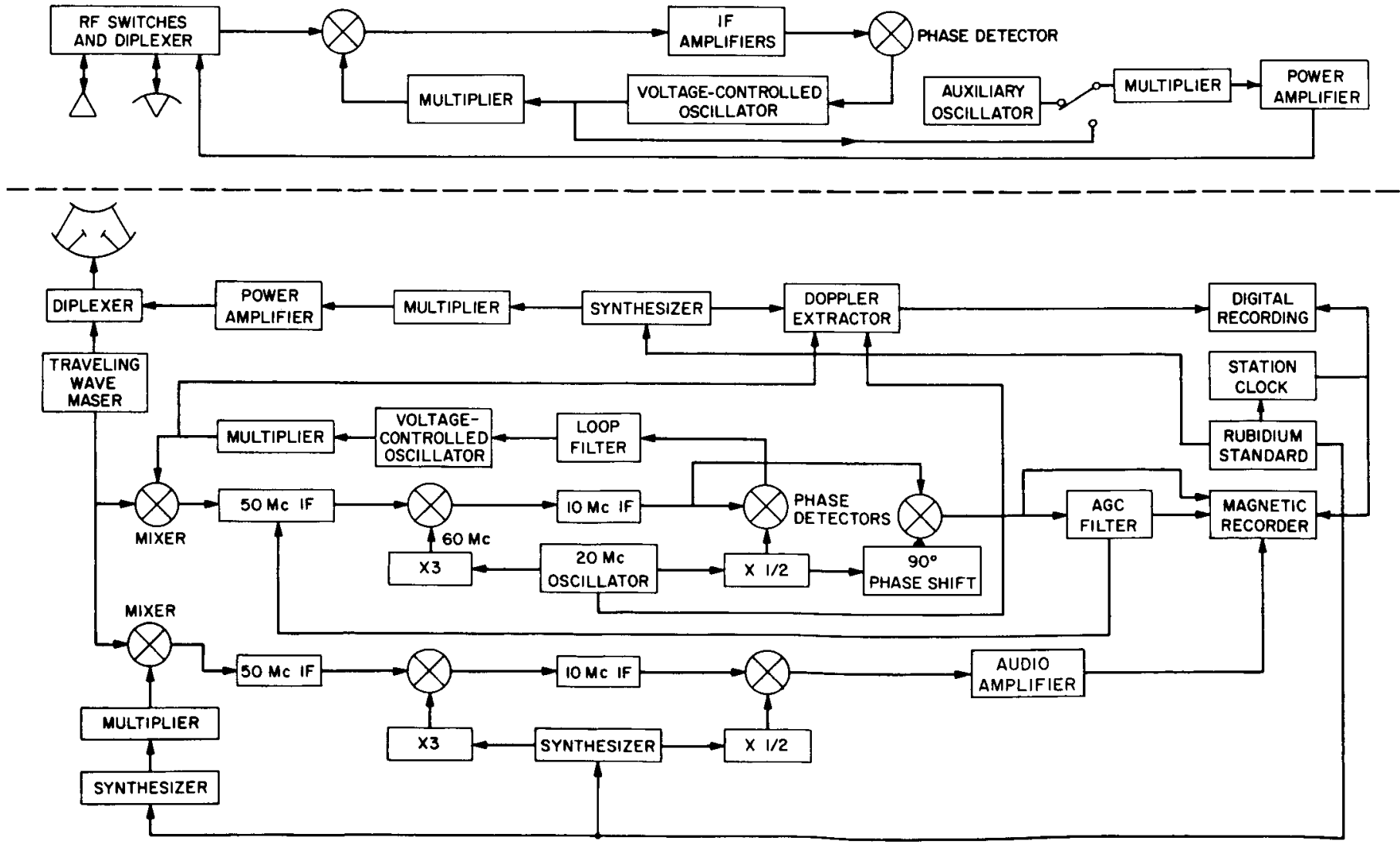

Fig. 3. Radio system for Mariner IV Occultation Experiment 


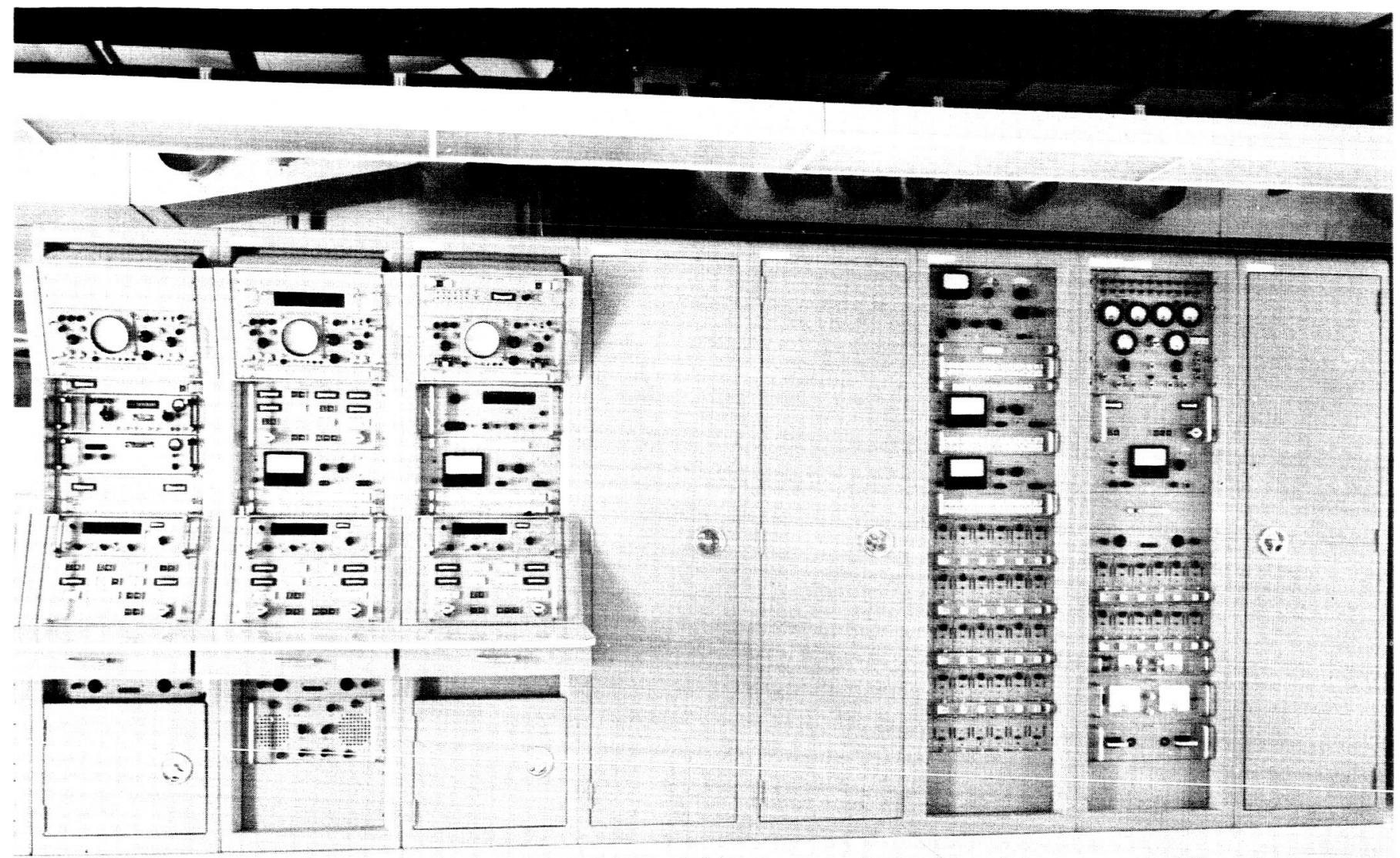

Fig. 4. DSIF phase-lock-loop receiver system

which is digitally cumulatively counted. The receiver automatic gain control (AGC), which is a receivedsignal power-level tracking servo, is used to determine received-power level. The output of this system is referred to as the static AGC voltage. Rapid changes in signal level can be determined from the dynamic $A G C$ voltage. The dynamic AGC is related to the AGC servo loop error signal (see Section III.A.2). The static and dynamic AGC voltages are recorded on both magnetic tape and chart recorder.

This receiver is also used as the sum channel for the simultaneous-lobing antemna tracking system.

\section{Open-Loop Occultation Receiver}

The second receiver channel is a manually tuned, constant-gain, triple-conversion superheterodyne operated in a nonstandard DSIF configuration. A photograph of instrumentation for this receiver may be seen in Fig. 5 . The receiver amplifies and frequency-translates the S-band input signal to the audio-frequency region of the spectrum. The local-oscillator ( $\mathrm{LO}$ ) signals for this receiver are derived from the rubidium frequency standard, which drives a pair of frequency synthesizers. The first synthesizer produces a signal of approximately $23.4 \mathrm{Mc}$ which is multiplied by 96 to produce the first LO at approximately $2.245 \mathrm{Gc}$. This LO frequency was periodically stepped to keep the signal in the receiver's pass band. The second and third LOs are derived from the second synthesizer, operating at $19.996 \mathrm{Mc}$. The output of the third mixer has a pass band of 1-3 kc, which is recorded on magnetic tape. The analog information on the magnetic tape is later digitized for use in a digital computer. An Electrac, Inc. ${ }^{1}$ model 215L audio phase-lock-loop tracking filter was used to provide a real-time indication of received signal being recorded. The open-loop receiver output was recorded on tape and then played back through a reproduce unit. The reproduced signal was fed into the Electrac tracking filter.

'Anaheim, California 


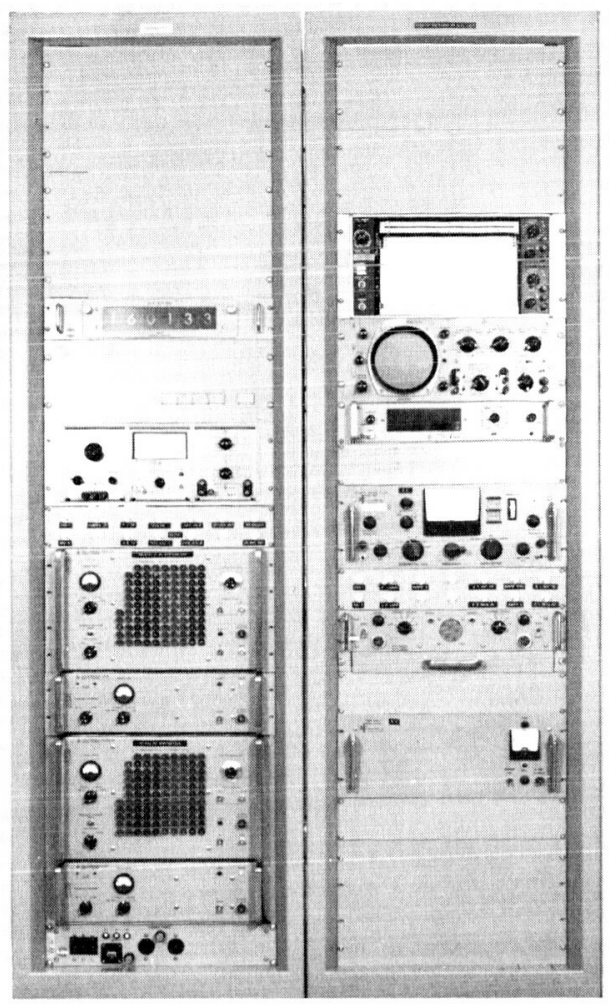

Fig. 5. Open-loop occultation receiver instrumentation

\section{Tape Recording Subsystem}

The DSIF tape recorder subsystem may be seen in Fig. 6. A typical FR 1200/100 recorder configuration used for recording data for the occultation experiment is shown in Fig. 7. Table $I$ shows typical tape recorder parametors ised. The recorders were set to record maximum anticipated signal voltages during the occultation phenomena.

To filter noise which was outside the frequency band of interest, the dynamic AGC voltage was filtered with a low-pass filter ( $3 \mathrm{db}$ at $50 \mathrm{cps},>40 \mathrm{db}$ above $100 \mathrm{cps}$ ). The phase and amplitude characteristics of the low-pass filter were measured before and after installation into the system.

Each tape reel used for recording during the occultation experiment was checked for dropout. Dropout is a term used to describe a sudden drop, due to imperfections of the tape, in the level of the signal recorded on the magnetic tape.

A prime requirement of the recording was that the frequency should not be distorted by more than a frac-

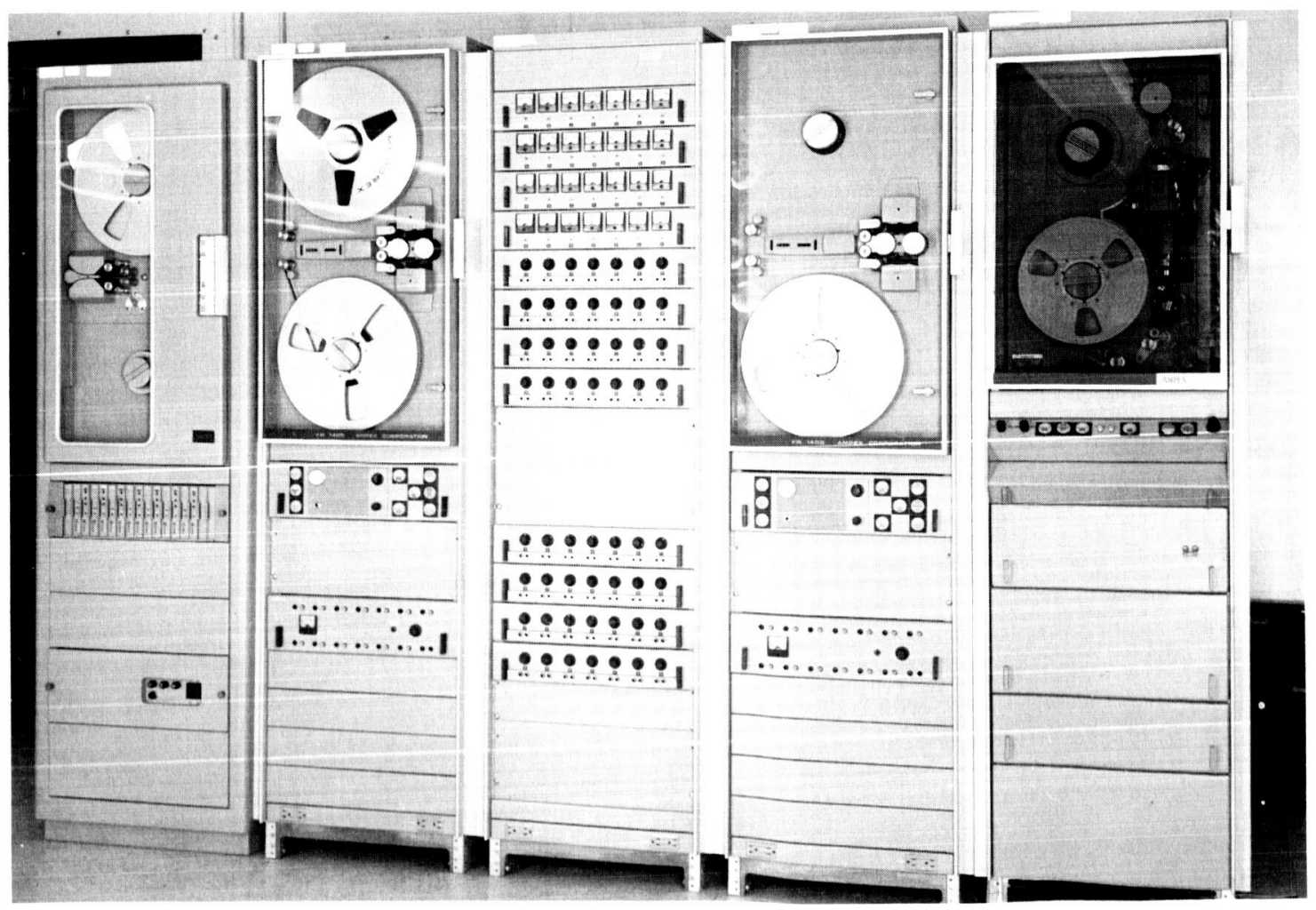

Fig. 6. DSIF tape recording subsystem 


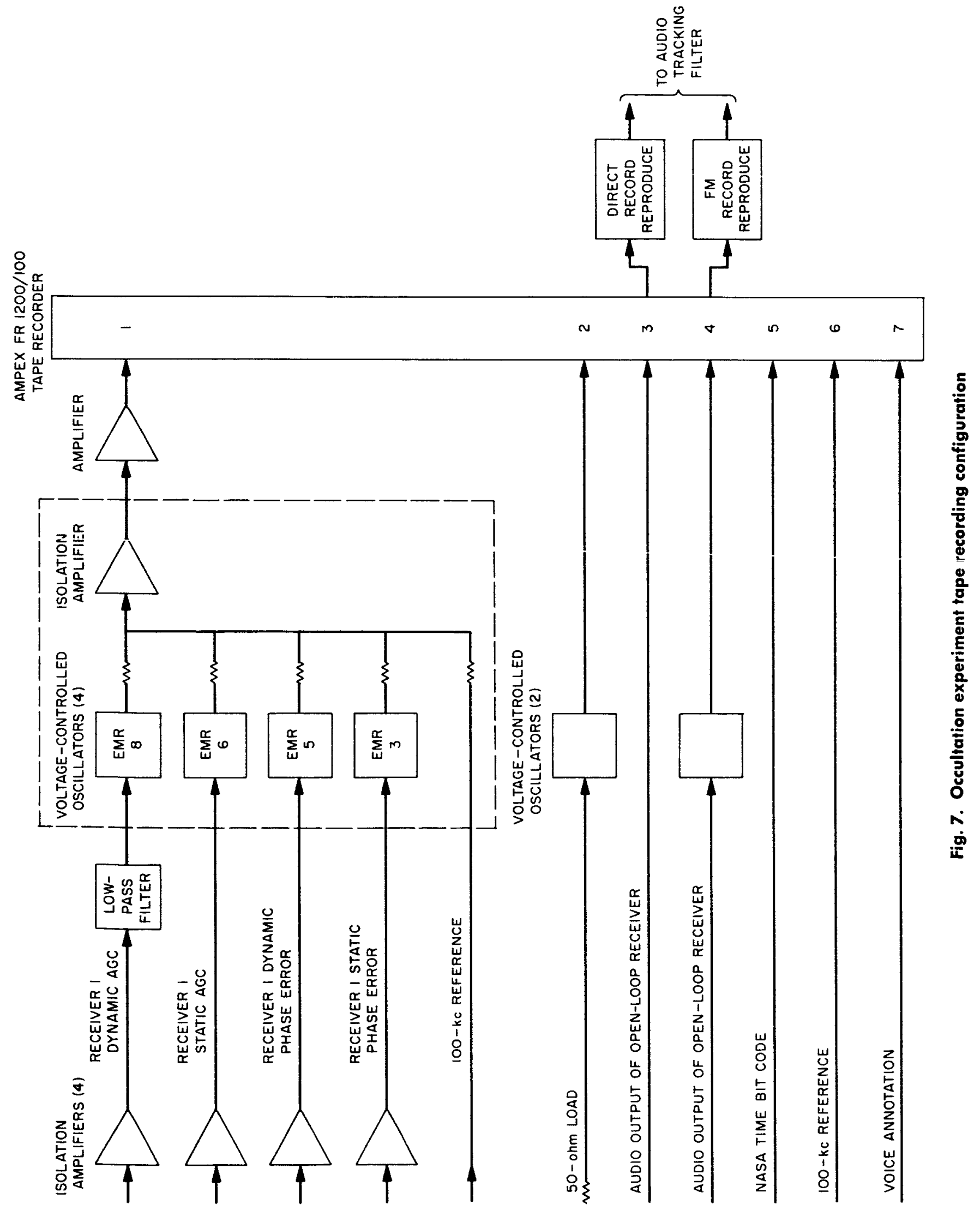


tion of a cycle per second. This was achieved in the recording band of 0.5 to $1.5 \mathrm{kc}$ by recording a frequency standard on an adjacent track and using it as a time base for keying the digitizer measuring the data channel. After digitization of the recordings, a digital computer was used to detect and analyze the signal. The result of this processing can yield both frequency and power as functions of time. The signal-to-noise ratio of the received signal is obtained by comparing the power spectral density of the broadband noise with the signal power.

As a result of tests made to determine optimum recording techniques, it was decided that FM tape recordings of the occultation phenomena be made with a recorder speed of 30 inches per second (ips). This speed was chosen because (1) the recording fidelity is better at the higher speeds and (2) this was the highest speed suitable for the continuous recording time required.

A discussion of preliminary tests and calibrations made on the standard DSIF phase-lock-loop receiver and the open-loop occultation receiver is given in the following Section. These tests were made to ensure satisfactory operation of the receiver systems during the occultation experiment and to develop techniques for accurate calibrations of the received signal.

Table 1. FR 1200/100 tape recorder track assignments

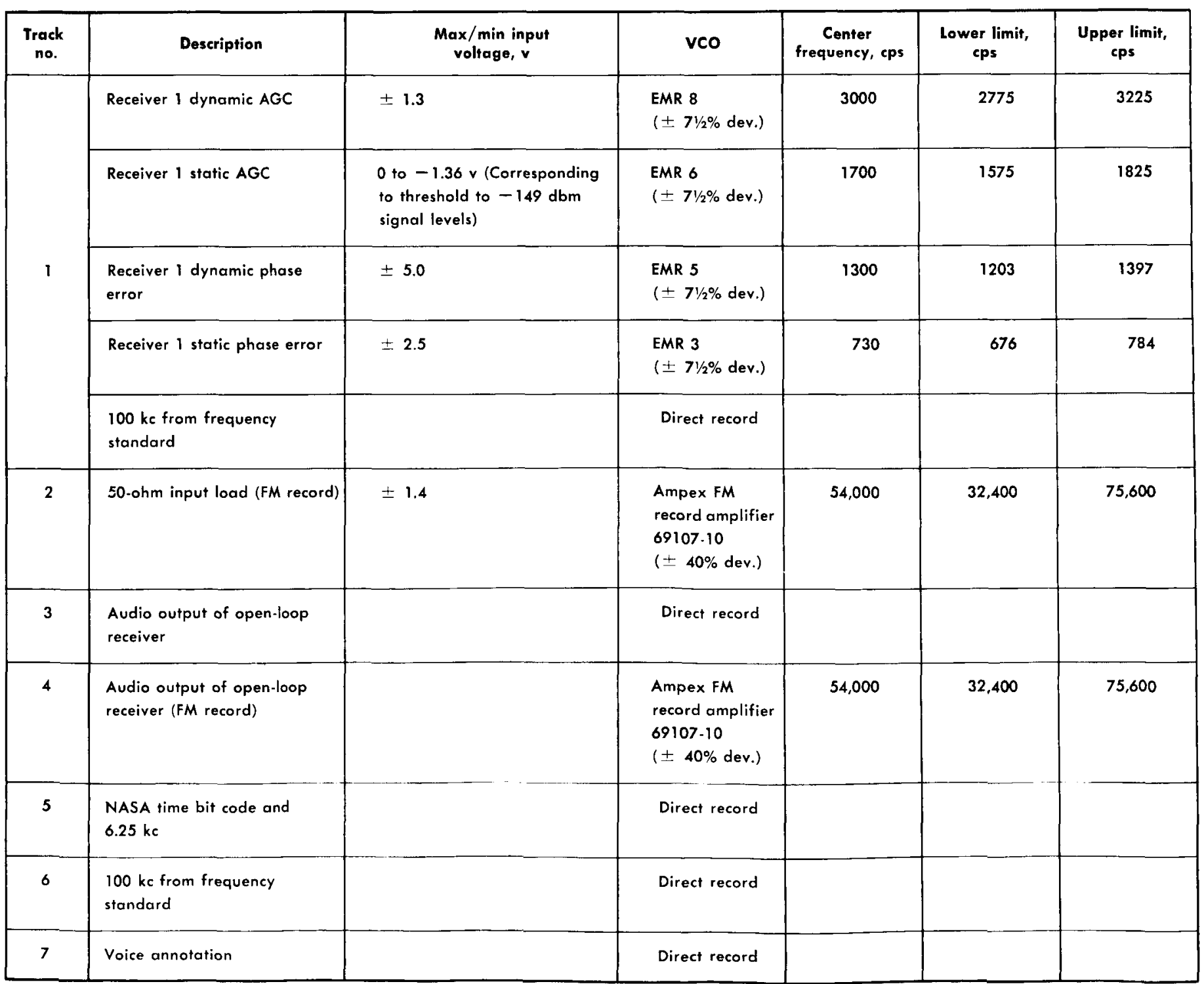




\section{RECEIVER AND SPACECRAFT SYSTEM TESTS}

\section{A. Phase-Lock-Loop Receiver Tests}

\section{Doppler Extraction Tests}

In the DSIF, doppler data are normally acquired over long periods of time. To minimize the quantization error, a cumulative count of biased doppler frequency is taken in which the total number of positive-going zero crossings are counted. The error for any one interrogation interval is \pm 1 count; however, the cumulative error is also \pm 1 count for any number of interrogation intervals. The doppler shift is obtained by taking the differences less the bias frequency of consecutive counts. The integral, or doppler phase, is obtained by taking a reference time and then obtaining the cycle count between the reference time and succeeding times less the bias frequency. The doppler phase has a greater inherent accuracy because any individual count difference has a quantization error of \pm 1 but the quantization error is not cumulative. In the occultation case, the highest frequency resolution possible was required. When the doppler frequency is multiplied by eigni before being counted, the quantization error is reduced and the resolution is increased.

A device was developed by Carl Johns of JPL to take the $1-\mathrm{Mc}$ offset doppler frequency from the doppler extractor, add an additional bias frequency, multiply it by eight, and subtract all the multiplied bias so that the output signal of the device is the RF doppler frequency multiplied by eight. This multiplied signal was then counted by the tracking data system.

The doppler multiplier was installed and tested at DSIF-11. Two Hewlett-Packard synthesizers were driven by the station rubidium standard. One synthesizer was used in the transmitter-synthesizer assembly, and the other was used to drive the test transmitter. By using two synthesizers, it was possible to simulate a very stable doppler offset. The data handling system performed 10-sec cumulative doppler counts. The standard deviation (rms) of the counts was reduced to cycles per second (cps) of doppler at S-band. The threshold was $-163 \mathrm{dbm}$. At $-145 \mathrm{dbm}$ the $\mathrm{rms}$ was 0.00738 , at $-155 \mathrm{dbm}$ it was 0.00892 , and at $-161 \mathrm{dbm}$ it was 0.18382 cps. After these measurements were made, a series of 1-sec cumulative-count measurements were made using the $\times 1, \times 2$, and $\times 8$ doppler counters. The $\times 2$ doppler counter uses the same input as the $\times 1$ but counts both positive- and negative-going zero crossings.
Figure 8 shows the relationship of the standard deviation of the 1-sec cumulative doppler count as a function of the input-signal level in $\mathrm{dbm}$. The upper curve was made using $\times 1$, and the lower curve using a $\times 8$ multiplier.

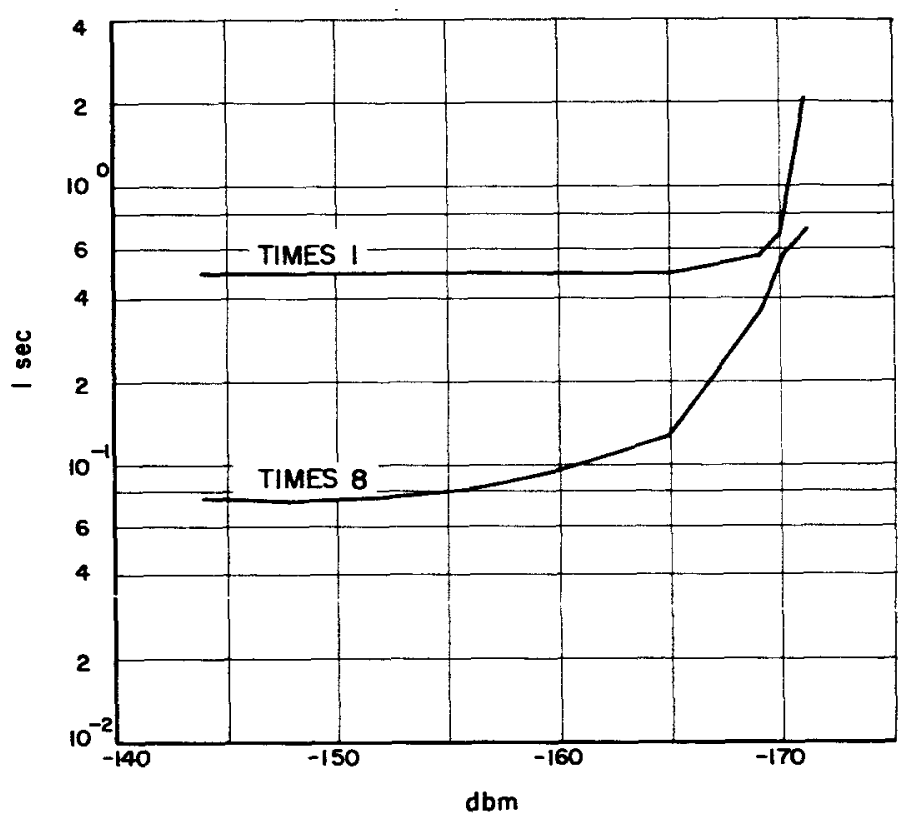

Fig. 8. One-second cumulative doppler standard deviation

\section{Closed-Loop AGC Tests}

A testing program was conducted to determine the required procedures for obtaining received-signal power, as a function of time, from the dynamic and static AGC.

a. Frequency response. One of the closed-loop tests made was a measurement of the AGC loop frequency response. A sinusoidally amplitude-modulated RF carrier was used as the receiver input signal and the AGC loop amplitude and phase responses were measured at the dynamic and static AGC outputs. These tests were made at a strong $\mathrm{RF}$ signal level (approximately $70 \mathrm{db}$ above threshold), with modulation frequencies from 0.01 to 100 cps. Figure 9 shows the equivalent AGC loop block diagram for dynamic signal conditions. Derivation of the theoretical equation for the AGC closed-loop transfer function is discussed by Victor and Brockman (Ref. 4). 


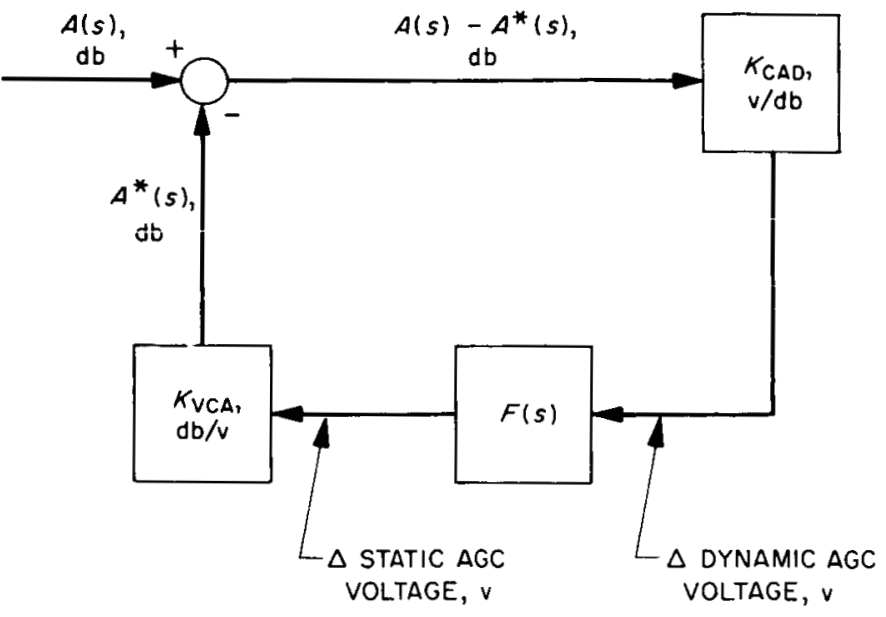

Fig. 9. Equivalent $A G C$ loop for dynamic signal conditions (noise-free case)

Using the equivalent AGC loop model given in Figure 9, we obtain the following AGC closed-loop transfer function

$$
H(s)=\frac{A^{*}(s)}{A(s)}=\left[\frac{K_{\mathrm{CAD}} K_{\mathrm{VCA}} F(s)}{1+K_{\mathrm{CA} 1} K_{\mathrm{VCA}} F(s)}\right]
$$

where

$$
\begin{aligned}
A(s)= & \text { Laplace transform of the input-signal ampli- } \\
& \text { tude change expressed in decibels (see dis- } \\
& \text { cussion, Ref. 4) }
\end{aligned}
$$

$$
A^{*}(s)=\text { Laplace transform of the resultant change in }
$$
receiver attenuation expressed in decibels

$$
\begin{aligned}
K_{\mathrm{CAD}}= & \text { coherent amplitude-detector gain parameter, } \\
& \mathrm{v} / \mathrm{db} \text { (approximately } 0.115 \mathrm{v} / \mathrm{db} \text { for the strong } \\
& \text { signal cases) }
\end{aligned}
$$

$$
\begin{aligned}
K_{\mathrm{vCA}} & =\text { receiver IF gain parameter, } \mathrm{db} / \mathrm{v} \\
F(s) & =\text { AGC loop filter transfer function } \\
s & =\text { complex frequency }
\end{aligned}
$$

If the AGC loop filter is an operational-amplifier phase-lag-inverting circuit, then the AGC loop filter transfer function is given by

$$
F(s)=\left[\frac{-G_{F}}{1+G_{F} \tau_{F} s}\right]
$$

where

$$
\begin{aligned}
G_{F} & =\text { ratio of the feedback and input resistors } \\
\tau_{F} & =\text { filter time constant, sec }
\end{aligned}
$$

By substituting the expression of $F(s)$ into Eq. (1) and letting $s=j \omega$ for the steady-state case of a sinusoidally modulated input, the closed-loop AGC response becomes

$$
H\left(j_{\omega}\right)=\left\lceil\frac{1}{\left(1-\frac{1}{G_{L}}\right)-i\left(\frac{\tau_{L \omega}}{G_{L}}\right)}\right\rfloor
$$

where

$$
\tau_{L}=\text { AGC loop time constant }=G_{F} \tau_{F}
$$

and

$$
\begin{aligned}
G_{L} & =K_{C A n} K_{\mathrm{VCA}} G_{\mathrm{F}} \\
\omega & =\text { angular frequency }=2 \pi f \\
f & =\text { modulation frequency, cps }
\end{aligned}
$$

From Fig. 9, the dynamic AGC transfer function is derived as

$K_{\mathrm{CAD}}[1-H(j \omega)]=-\frac{K_{\mathrm{CAD}}}{G_{L}}\left[\frac{1+j \omega \tau_{L}}{\left(1-\frac{1}{G_{L}}\right)-i\left(\frac{\omega \tau_{L}}{G_{L}}\right)}\right]$

and the static AGC transfer function as

$$
\frac{1}{K_{\mathrm{VCA}}}[H(j \omega)]=\frac{1}{K_{\mathrm{rCA}}}\left[\frac{1}{\left(1-\frac{1}{G_{L}}\right)-i\left(\frac{\omega \tau_{L}}{G_{L}}\right)}\right]
$$

Using

$$
\begin{aligned}
K_{\mathrm{CAD}} & =+0.115 \mathrm{v} / \mathrm{db} \\
K_{\mathrm{VCA}} & =-16.7 \mathrm{db} / \mathrm{v} \text { (determined experimentally) } \\
G_{F} & =+100 \\
G_{L} & =K_{\mathrm{CAN} 1} K_{\mathrm{VCA} A} G_{F} \simeq-190 \\
\tau_{L} & =G_{F} \tau_{F} \simeq(100 \times 3.8)=380 \mathrm{sec}
\end{aligned}
$$


as the values for the theoretical parameters in Eqs. (4) and (5), the theoretical phase and normalized amplitude responses were calculated and are shown plotted in Figs. 10 and 11, for dynamic and static AGC, respectively. As may be seen in Figs. 10 and 11, the theoretical and measured amplitude and phase responses are in reasonably good agreement. The normalized amplitude and phase response at the output of the dynamic AGC for manual gain control (MGC) operation (open AGC loop) is also shown in Fig. 10. For MGC operation, the MGC voltage was adjusted to give the same IF receiver gain as that obtained during AGC operation. The estimated accuracy of the measured phase angles is $\pm 5 \mathrm{deg}$ for the pass-band frequencies.

b. Dynamic AGC calibrations. The ac component of the dynamic AGC voltage can be used to determine RF amplitude changes provided that the amplitude change is too rapid to be corrected by the servo action of the AGC loop. As shown in Fig. 10, when the receiver
RF loop bandwidth is set at $12 \mathrm{cps}$, and the AGC loop bandwidth is set at $0.118 \mathrm{cps}$, the 3-db lower limit occurs at $0.08 \mathrm{cps}$. The upper-frequency amplitude and phase characteristics are governed by the characteristics of a $\mathbf{1 0 - M c}$ filter in the receiver system.

Calibration of the dynamic AGC voltage was performed by a static calibration procedure. This procedure was verified under dynamic conditions using sinusoidal modulated RF signals whose modulation indices were known. The static calibration procedure was as follows:

At a specified RF signal level, the AGC voltage was noted and recorded. Then the receiver was operated in the MGC mode. In this mode of operation, the AGC feedback loop is open and the receiver gain is determined by the MGC voltage. The MGC voltage was then set equal to the AGC voltage previously recorded. The receiver was now operating with the same overall gain in MGC operation as it was for AGC operation at the

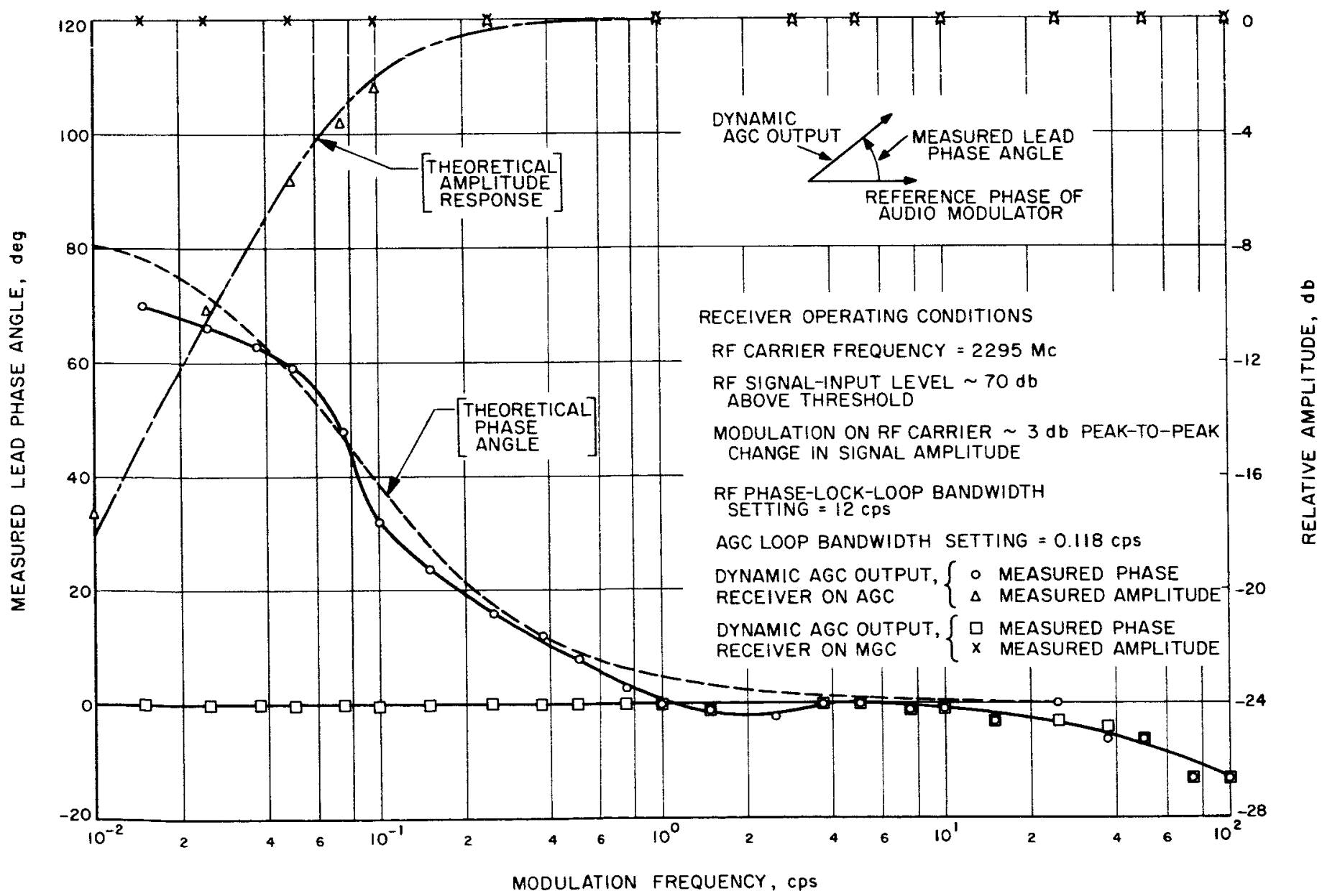

Fig. 10. Dynamic $A G C$ frequency response 


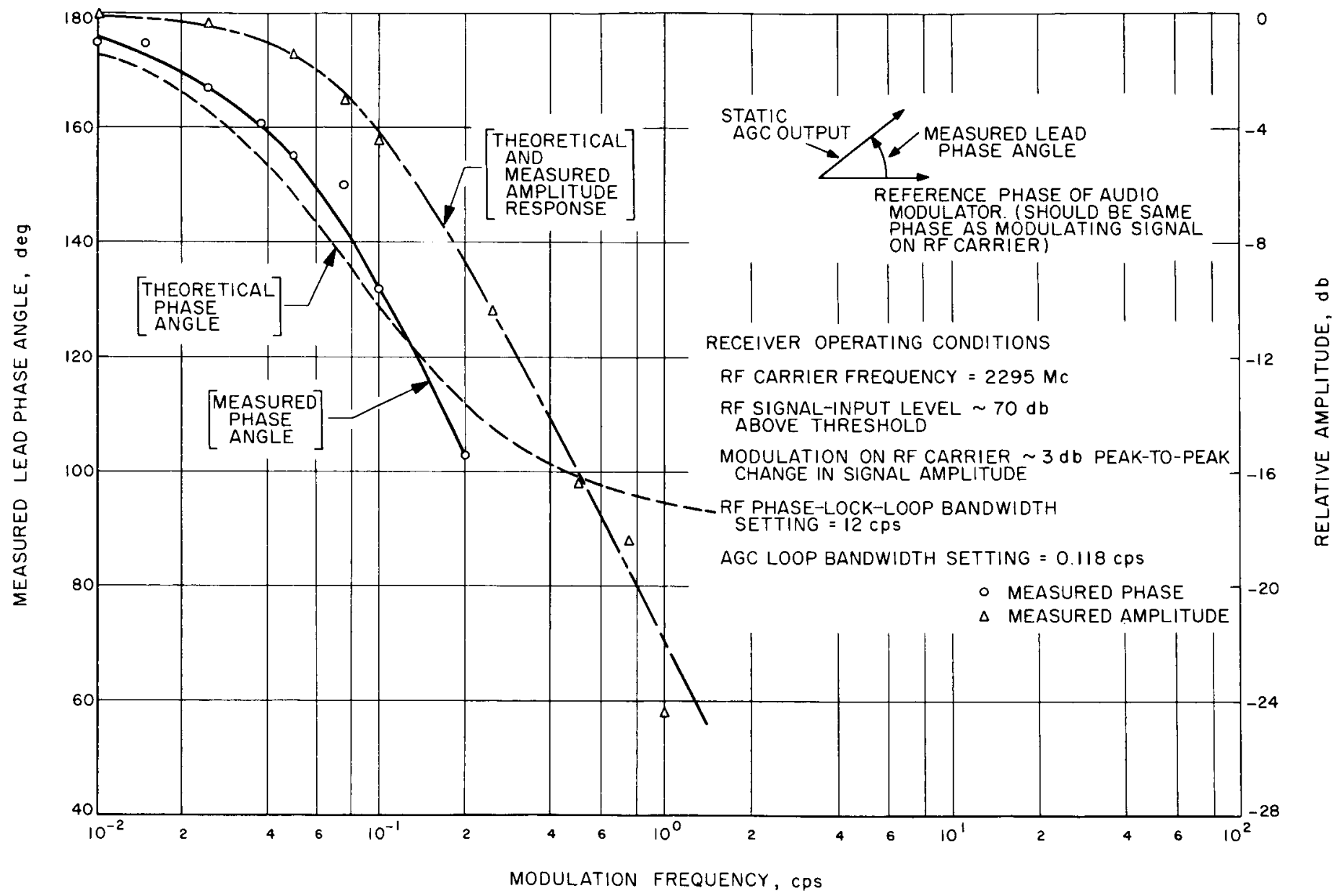

Fig. 11. Static AGC frequency response 
specified signal level. The RF signal level was then precisely changed by known amounts with a calibrated RF attenuator on the test transmitter.

The dynamic AGC voltage at the output of an isolationamplifier was fed into the Digital Instrumentation Subsystem (DIS) computer. The computer sampled the input voltage at a rate of $100 \mathrm{samples} / \mathrm{sec}$ for $10 \mathrm{sec}$ and then computed the mean dynamic AGC voltage which was printed out on a line printer. A typical example of the results of this calibration procedure may be seen in Fig. 12. Each data point in Fig. 12 was obtained by averaging the output data for $3 \mathrm{~min}$ or more. It may be seen that the output of the receiver is linear with respect to the RF signal voltage. The slopes of these curves change slightly at different reference signal levels.

c. Static AGC Calibrations. The static AGC voltage is normally used at DSIF tracking stations to determine average RF signal level. Calibration is performed before and after each tracking period.

The signal strength is determined from the output power of a calibrated RF transmitter corrected for the calibrated losses from the test transmitter to the input to the maser. The calibrated RF attenuator on the test trans- mitter is varied to change the signal strength by known amounts. AGC voltage versus received-signal level is illustrated in Fig. 13.

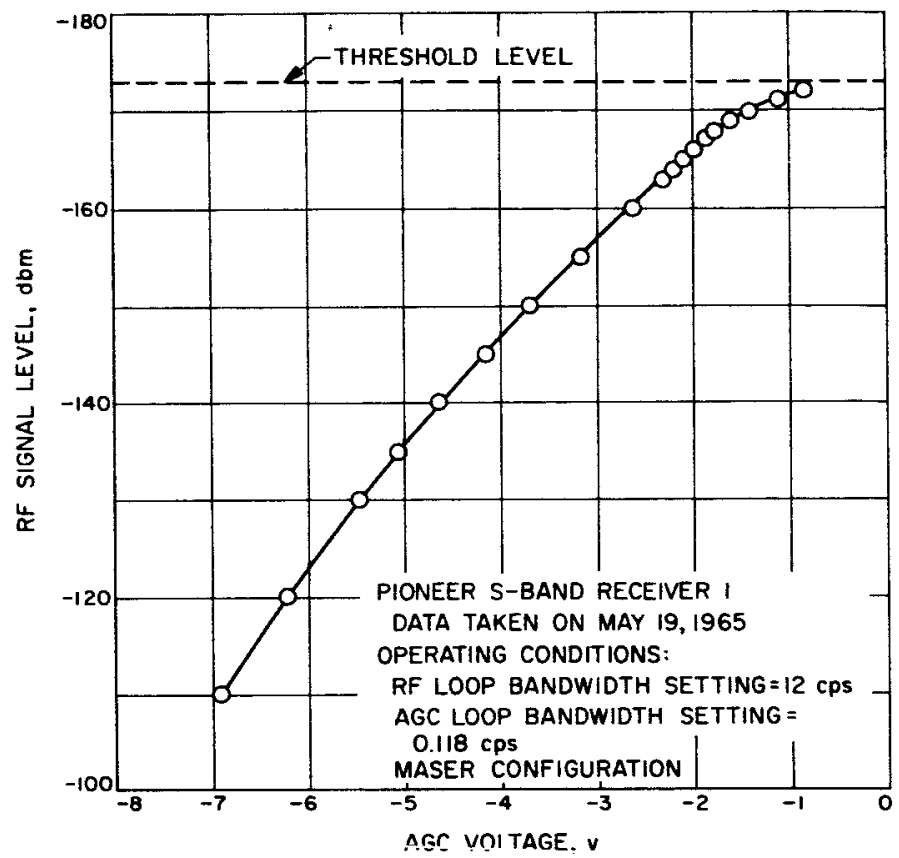

Fig. 13. AGC characteristic curve

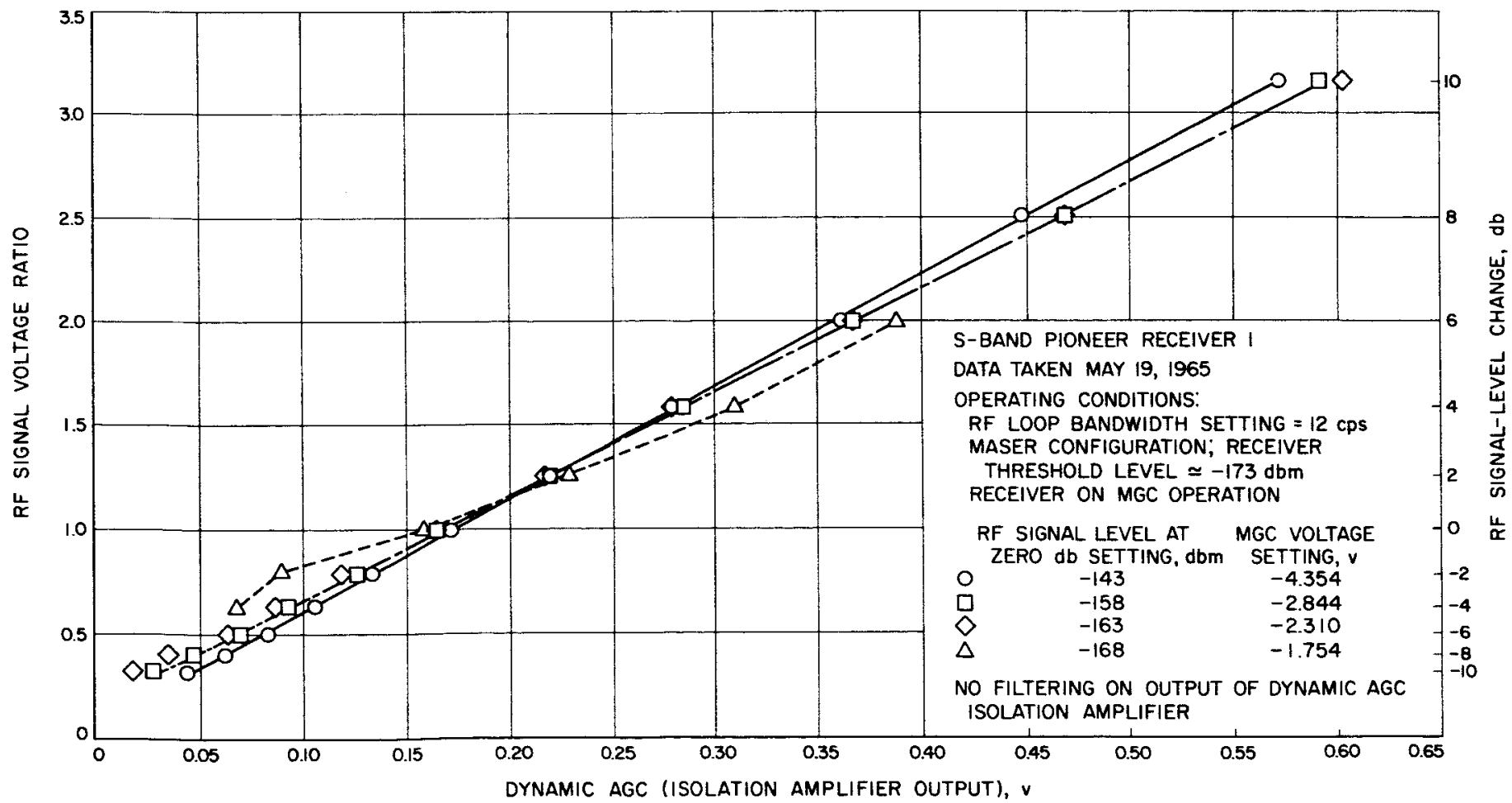

Fig. 12. Normalized RF signal voltage vs dynamic AGC voltage 


\section{B. Open-Loop Occultation Receiver Tests}

\section{Signal Level Calibrations}

Signal level calibrations of the open-loop receiver were performed simultaneously with the closed-loop receiver AGC calibrations. The test-signal level at the input to the maser is the same for hoth open and closed-loop receivers. The test-signal level was changed in known amounts by the calibrated RF variable attenuator on the test transmitter. The audio output voltage of the openloop receiver was calibrated in terms of the RF signal level.

\section{Spectral Analyses}

On the night of April 1, 1965 the open-loop receiver channel was used to receive the Mariner IV signal. The parametric amplifier was employed so that the signal in the phase-lock-loop was approximately $14 \mathrm{db}$ above threshold (roughly the level expected at the encounter). The output of the open-loop receiver was recorded on magnetic tape. A spectral analysis was made later at JPL using an analog technique. Figure 14 shows a power spectral density plot in which a 24.6-cps window was employed; power is plotted on a logarithmic scale. In Fig. 15, a 6.2-cps window was used to obtain a plot that is proportional to power. The higher-order harmonics of the phase-modulated signal are clearly discernible in Fig. 15. The above data were recorded using a direct magnetic recording technique at a tape speed of $33 / 4$ ips.

\section{Spacecraft Transponder Acquisition-Time Tests}

As the spacecraft emerged from behind the planet, an attempt was made to lock the vehicle transponder to the uplink. It was desired that the transponder be locked to the uplink because the frequency stability is several orders of magnitude greater in the locked condition than when it is being driven by the auxiliary oscillator. However, the drawbacks of attempting transponder lockup were due to the uncertainties of lockup time and the attendant frequency and power transients which occur when the vehicle switches from the auxiliary oscillator to the uplink signal.

To determine the lock-on times to be expected, a series of tests were performed in an environmental vacuum test tank on the type approval vehicle radio system. A synthesizer was driven by a rubidium standard. The output of the synthesizer was multiplied up in the test transmitter, attenuated, and sent into the transponder in the

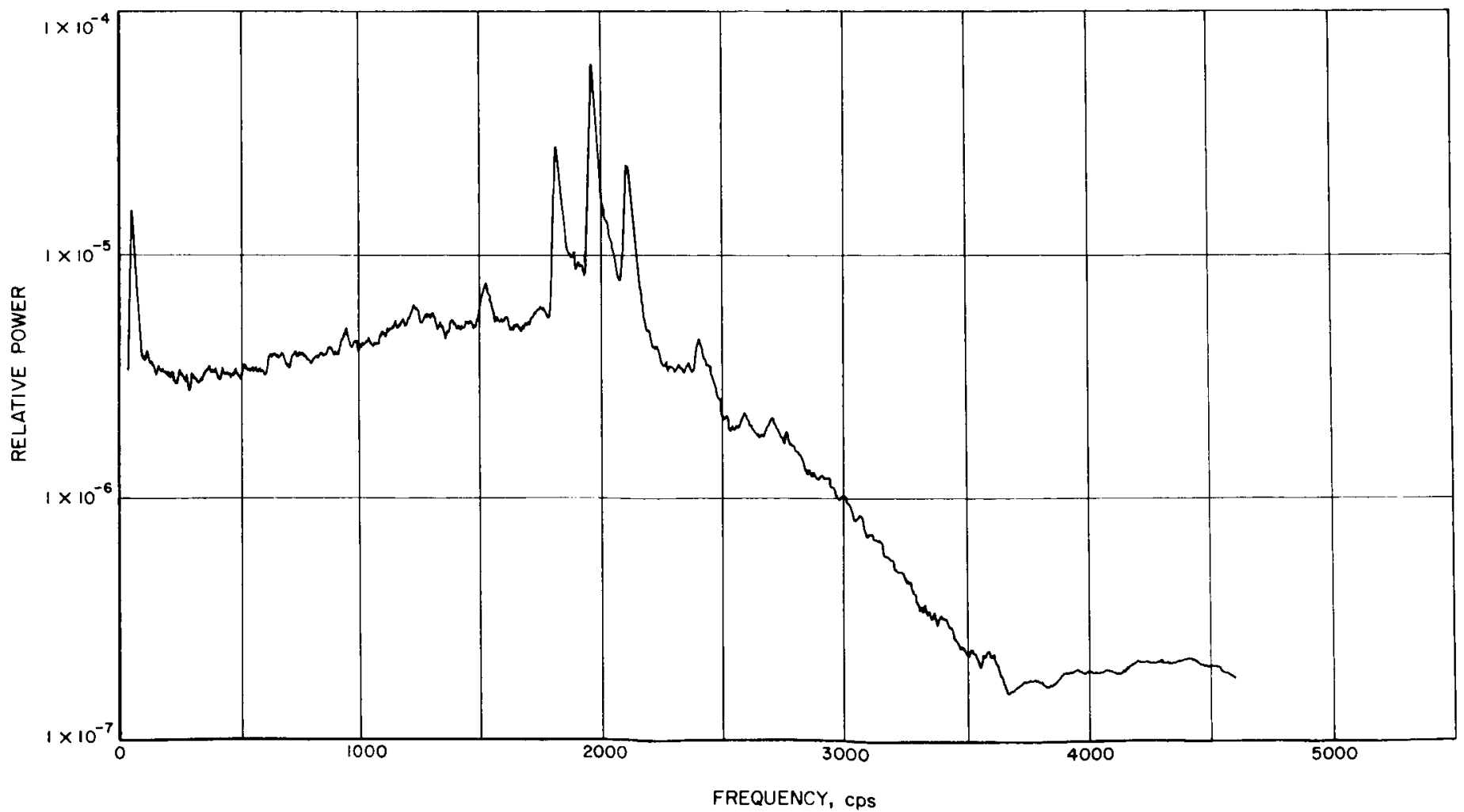

Fig. 14. Power spectral density of Mariner IV signal using 24.6-cps window 


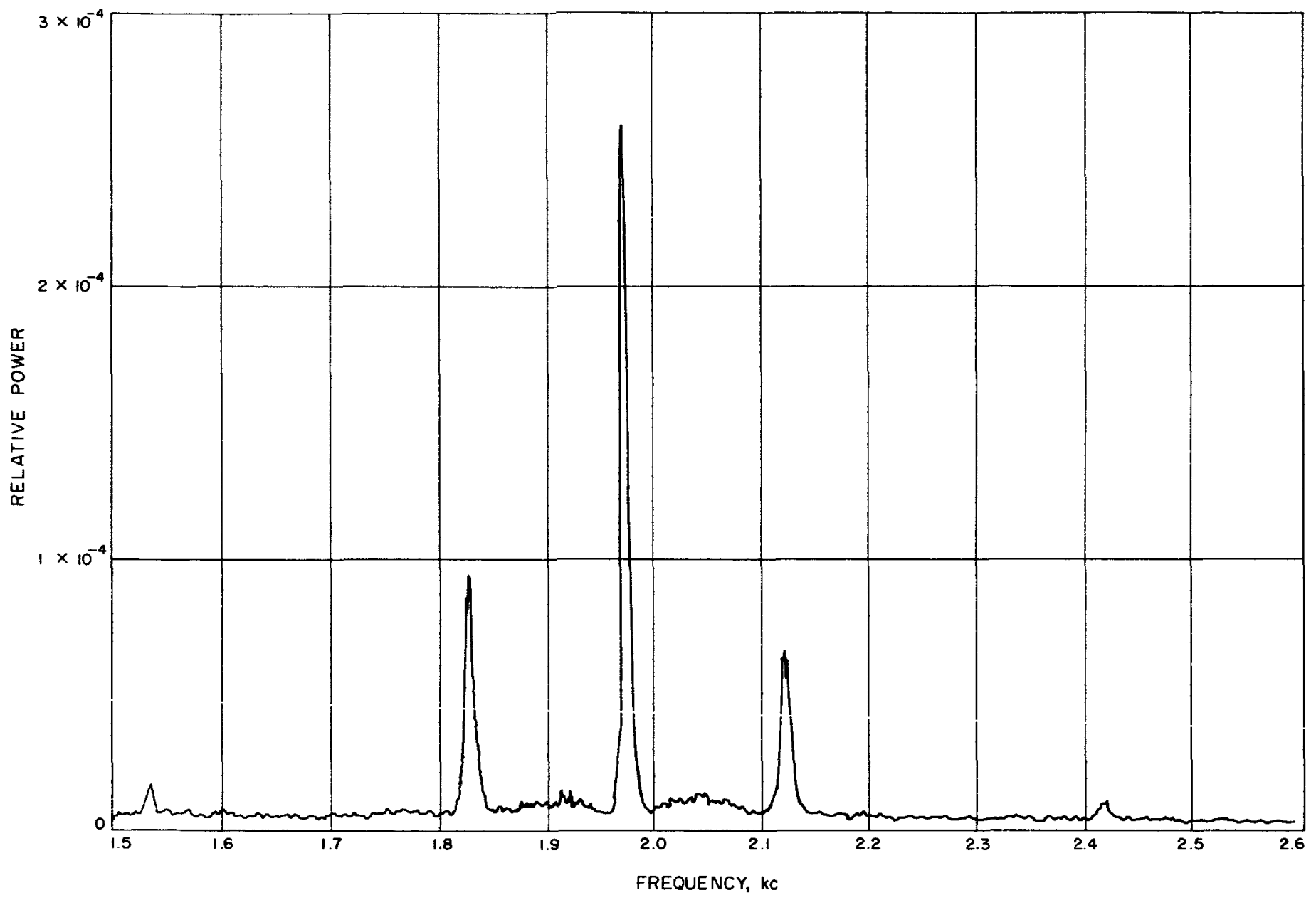

Fig. 15. Power spectral density of Mariner $I V$ signal using 6.2-cps window

environmental test chamber. The output of the transponder was monitored for frequency and power level. The AGC voltage and the voltage on the switch used to select either the auxiliary oscillator or the VCO, were also monitored. They were found to give an excellent indication of the time of switching. A digital datalogging system was used to record the transponder output data as well as the transmitter on-off status on magnetic tape.

Figure 16 is a plot of mean lock-on times as a function of offset from best-lock frequency. There are three curves: $-120,-130$, and $-140 \mathrm{dbm}$. The low-gain vehicle antenna in conjunction with the $100 \mathrm{kw}$ transmitter, produces a spacecraft received-signal level of approximately $-124 \mathrm{dbm}$ without command modulation, or $-127 \mathrm{dbm}$ with command modulation. It should be emphasized that these measurements have a very large statistical spread, and the averages for each point on the graph were taken from only 3 to 16 measurements. For example, the mean lock-on time for $-130 \mathrm{dbm}$ at no frequency offset is $1.7 \mathrm{sec}$; however, the minimum value was $0.6 \mathrm{sec}$, and the maximum was $2.7 \mathrm{sec}$ out of a total of 16 measurements. According to Viterbi's analysis (Ref. 5) for a noise-free case, at best-lock frequency the acquisition time goes to zero. However, in the receiver a best-lock frequency does not exist because of noise; but there is, rather, a best-lock frequency probability distribution function.

The VCO output was measured when the receiver was out of lock. This was done on the serial number 7 transponder system. It can be seen in Fig. 17 that the histogram has the shape of a noisy normal distribution.

Before multiplication by 120 , the frequency was measured so that the standard deviation of the receiver out of lock is $1.32 \mathrm{kc}$ at S-band. It must be remembered, however, that these measurements have not been made on the spacecraft itself but, rather, on similar equipment 


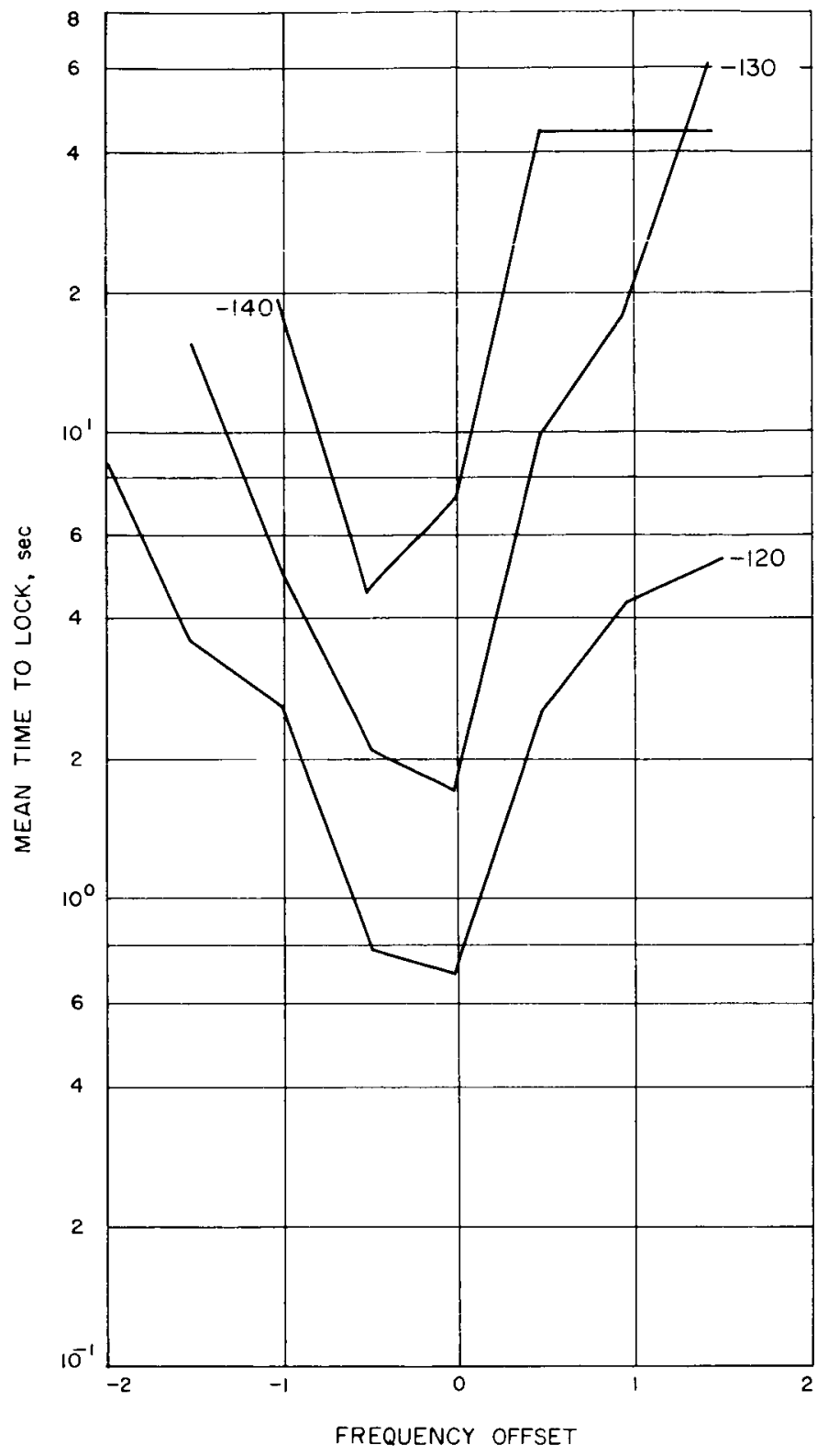

Fig. 16. Transponder lock-on times vs frequency offset

in a space simulation. A further complication at encounter arose from the temperature transients that were induced by turning on the encounter-mode experiments, which caused an additional perturbation on the VCO frequency.

\section{Simulation of Expected Amplitude Modulation}

An important test made on both open-loop and closedloop receivers was to calibrate receiver response to an $\mathrm{RF}$ input signal which was amplitude modulated to simulate Fresnel diffraction theory (Fig. 2).

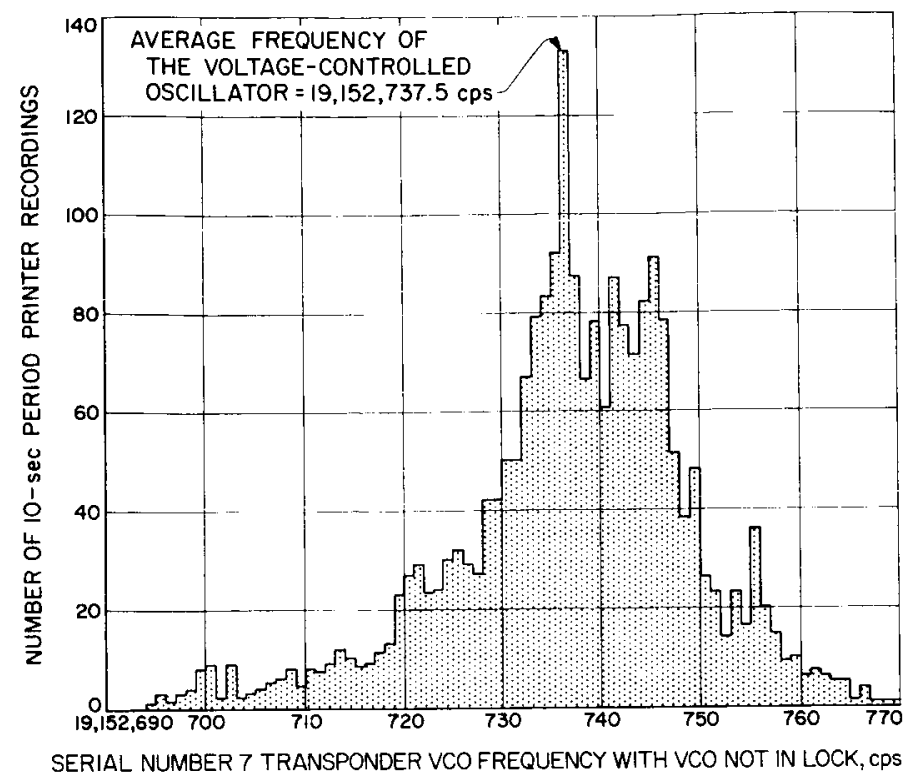

Fig. 17. Number of 10-sec counts vs VCO frequency when not in lock

To adequately simulate the diffraction phenomena, an IBM 1620 program was written to synthesize the voltage function necessary to drive an RF modulator to produce the expected Fresnel effect. The punched-card output of this program was then used on the digital PDP4 computer to obtain a digital-to-analog conversion. The ana$\log$ function was recorded on magnetic tape, which was then used on the FR 1400 tape recorder along with the appropriate amplification and biasing equipment to drive a Hewlett-Packard $8714 \mathrm{~A}$ modulator. (This modulator was biased to a region which was linear over a 6-db range.) The modulator was placed between the test transmitter and the receiver input.

Figure 18 shows the simulation recording of the amplitude fluctuations for a free-space signal level of -120 $\mathrm{dbm}$. The top trace is the modulator drive; the second is the dynamic AGC of receiver 1 in the manual gain control (MGC) position. A 50-cps low-pass filter is used at the dynamic AGC isolation-amplifier output. The third trace is derived from the open-loop receiver where the signal has been put through a 10 -cps band-pass filter prior to detection.

In Figure 19, the configuration is the same as the configuration for Figure 18 except that receiver 1 is in AGC. Figure 20 has an input-power level of $-158 \mathrm{dbm}$ and receiver 1 is in MGC. Figure 21 also has an inputpower level of $-158 \mathrm{dbm}$ and receiver 1 is in AGC. 


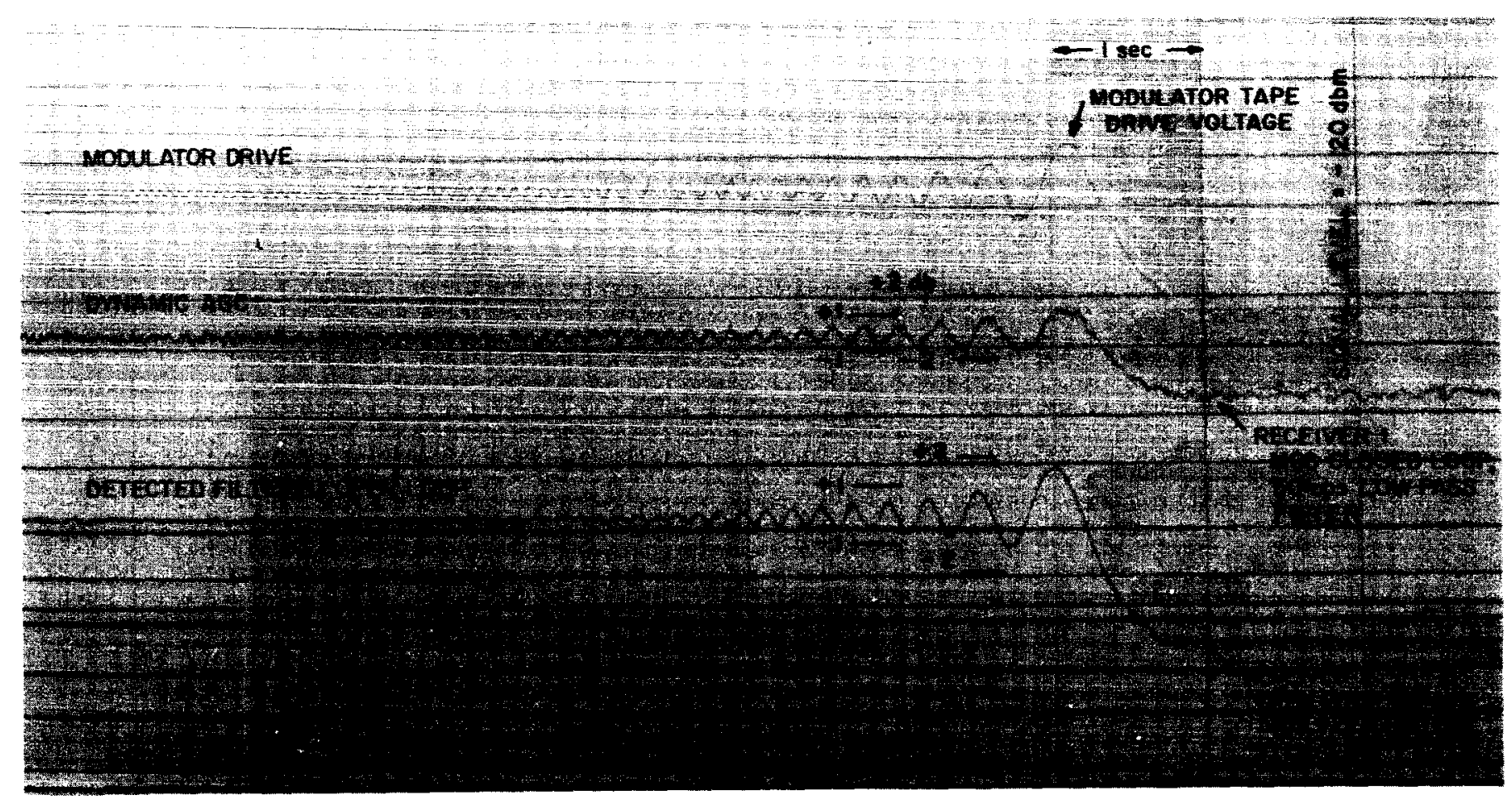

Fig. 18. Occultation simulation, input power - î̃û dbm, receiver i in MGC mede

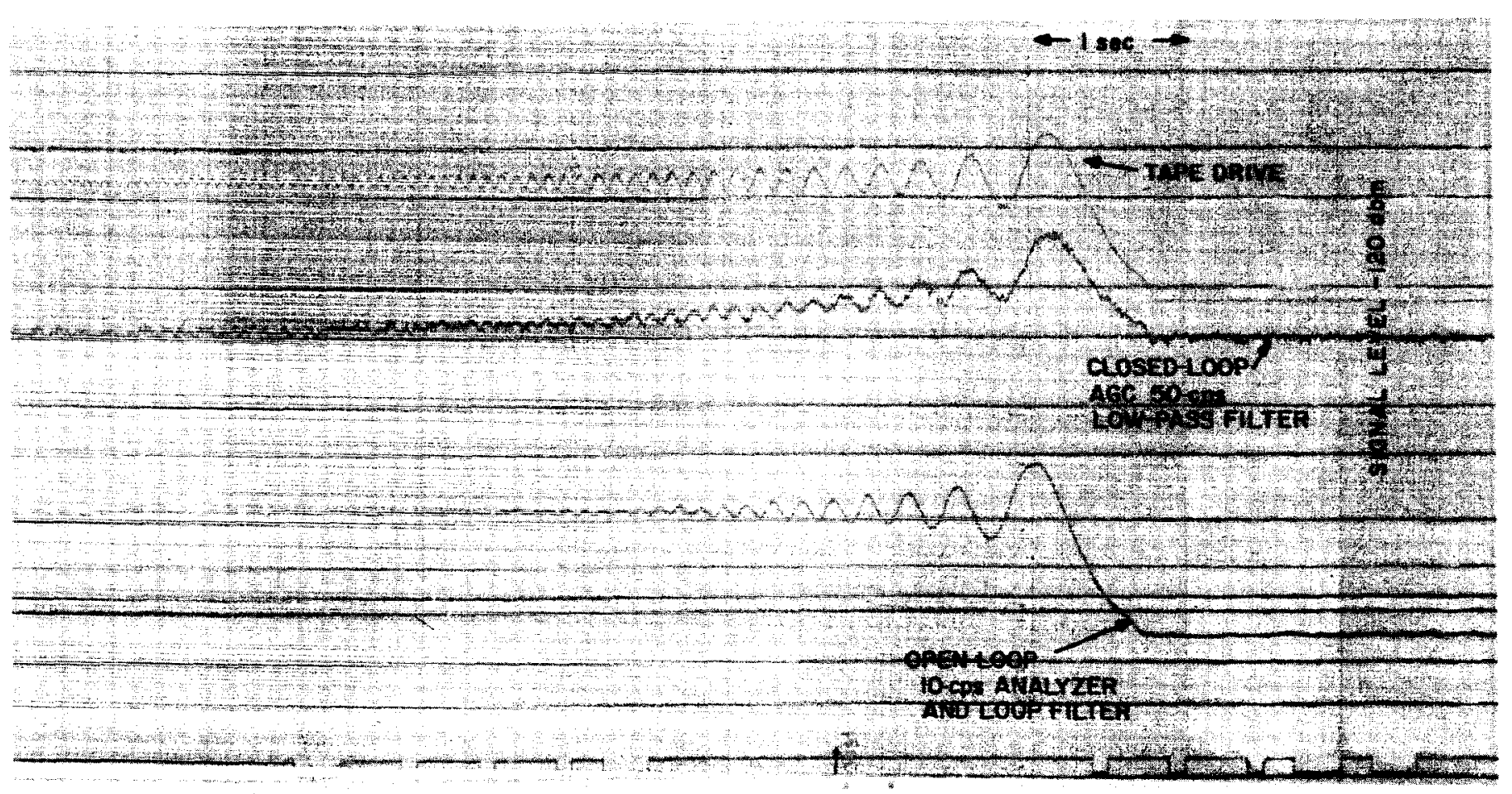

Fig. 19. Occultation simulation, input power $-120 \mathrm{dbm}$, receiver 1 in AGC mode 

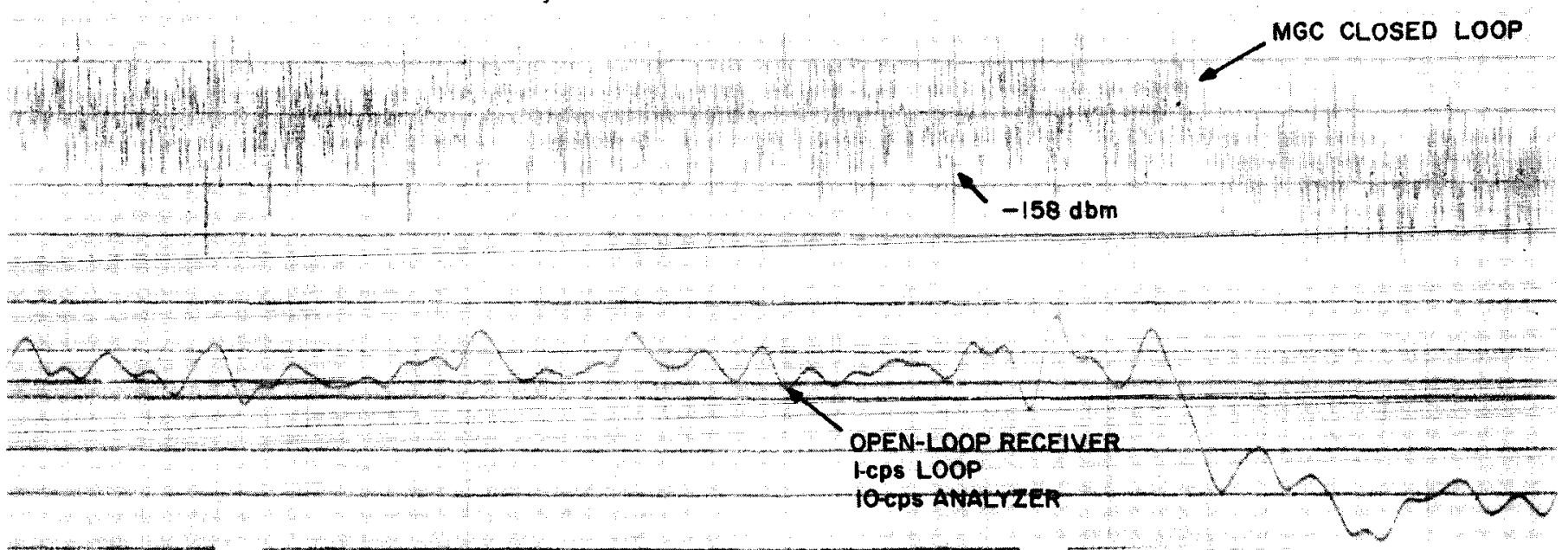

Fig. 20. Occultation simulation, input power $-158 \mathrm{dbm}$, receiver 1 in $M G C$ mode

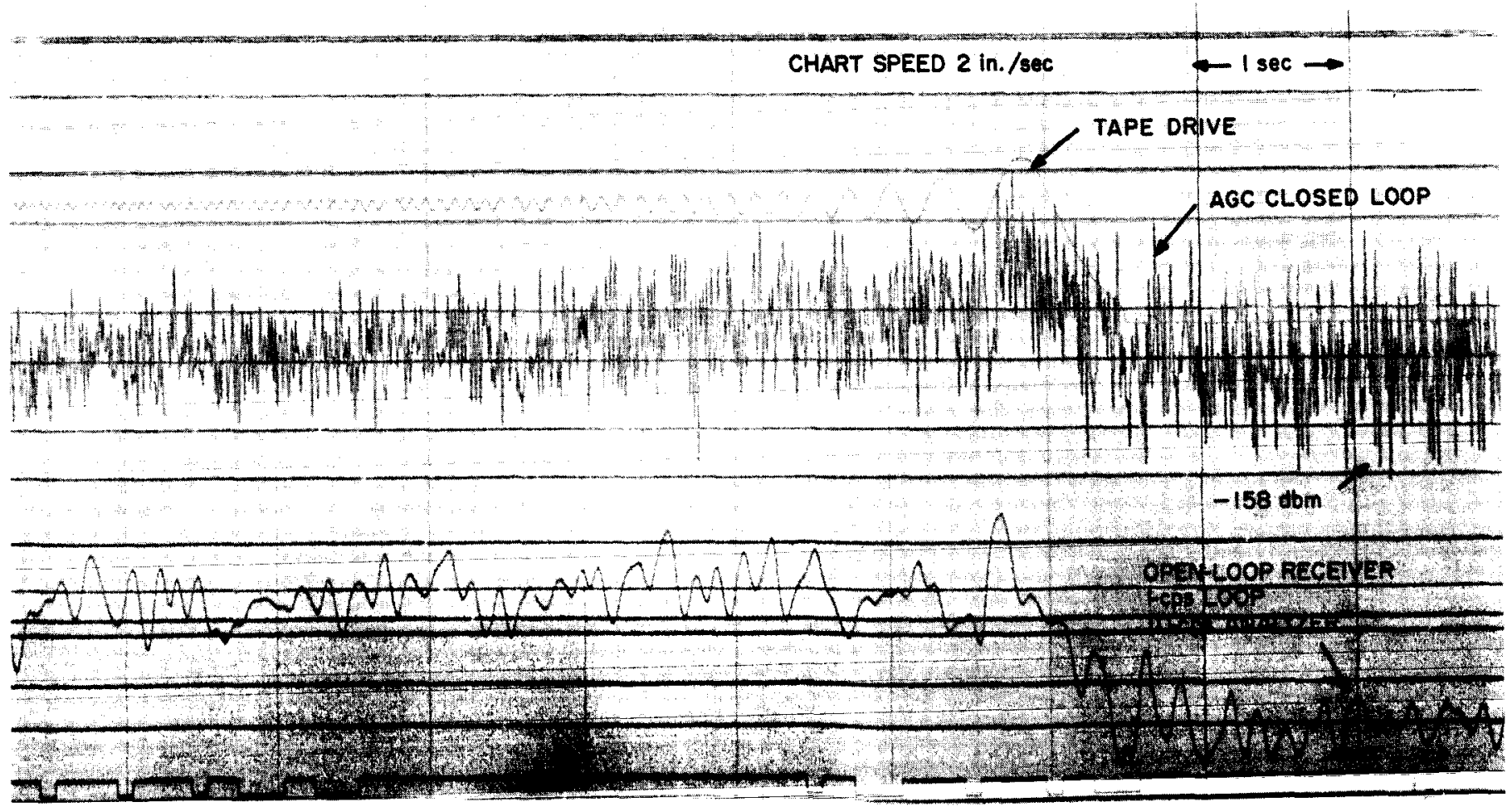

Fig. 21. Occultation simulation, input power $-158 \mathrm{dbm}$, receiver 1 in $A G C$ mode 


\section{RESULTS OF THE MARINER IV OCCULTATION EXPERIMENT}

The $\times 8$ doppler extractor output was digitized and relayed from Goldstone to JPL. The normal doppler data were also obtained from DSIF-42 in Australia. These actual doppler data were compared with the values predicted from the orbit-determination program. The doppler residuals were then taken to be the perturbations produced by the Martian atmosphere. Figure 22 shows the doppler residuals as a function of time obtained on closed-loop receivers as Mariner IV entered occultation. Figure 23 shows the doppler phase residuals as a function of time. The interpretation of these data is given in Refs. 2, 6, and 7 .

The open-loop signal was recorded at DSIF-11 and - 12 on FR 1200/100 tape recorders. The discriminated FM signal was later reduced at JPL. The recorded $100-\mathrm{kc}$ signal is used to minimize the effects of flutter. The 100 -kc signal was scaled down to $10 \mathrm{kc}$ and used to trigger the digitizer which digitized the discriminated FM signal.

The digitized signal was then processed on the IBM 7094. A broad-band spectral analysis was made for each 1-sec increment; Fig. 24 shows a broad-band spectrum made from the DSIF-12 tape. Although the resolution of this spectrum is quite coarse, it is adequate to determine the approximate audio frequency of the carrier. A digital band-pass program was then used. ${ }^{1}$ This program not only filtered out a portion of the noise but also permitted a significant reduction of the number of points to be handled in further processing. The filtering process also removed the telemetry sidebands.

${ }^{3}$ The technique was originated by Dr. S. Zohar, JPL.

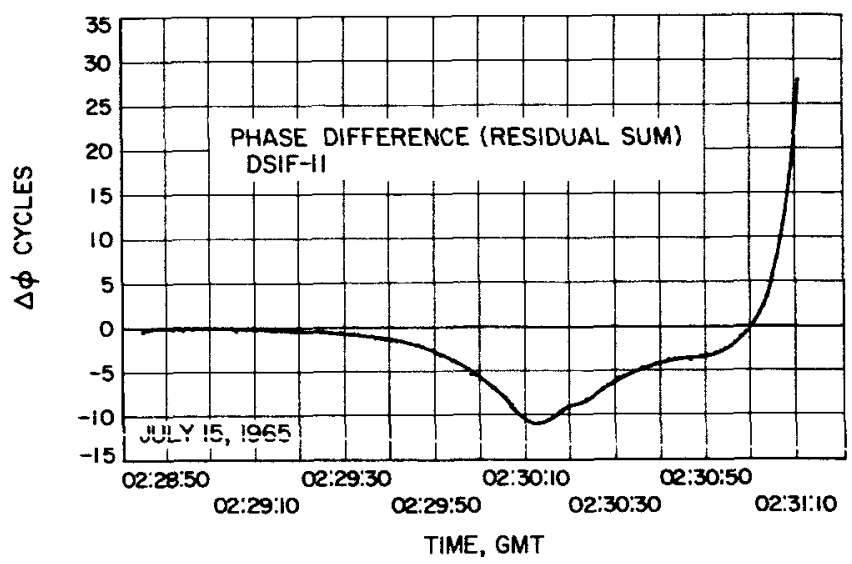

Fig. 23. Entry residual sum, closed loop

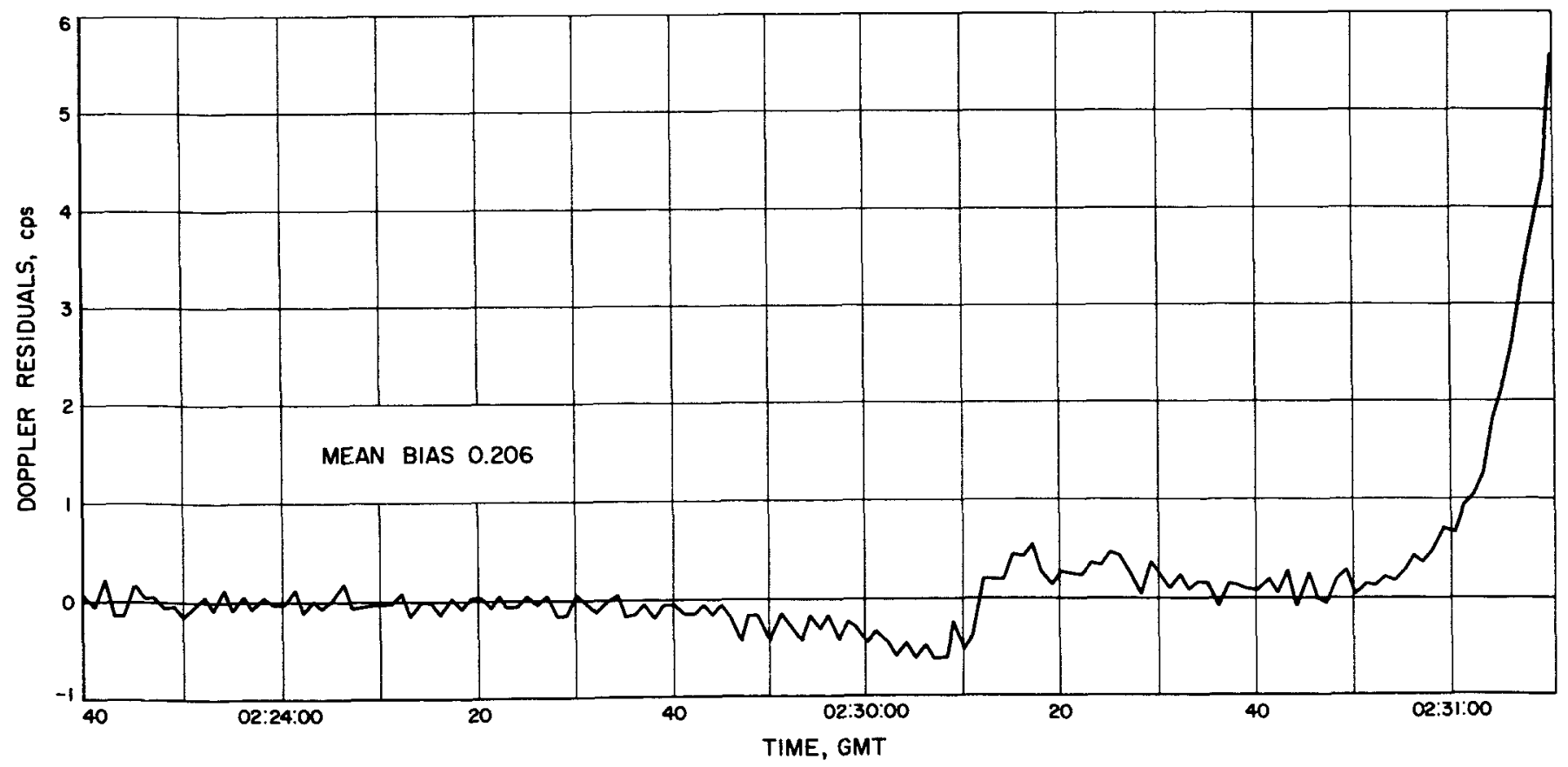

Fig. 22. Entrance occultation closed-loop doppler residuals 


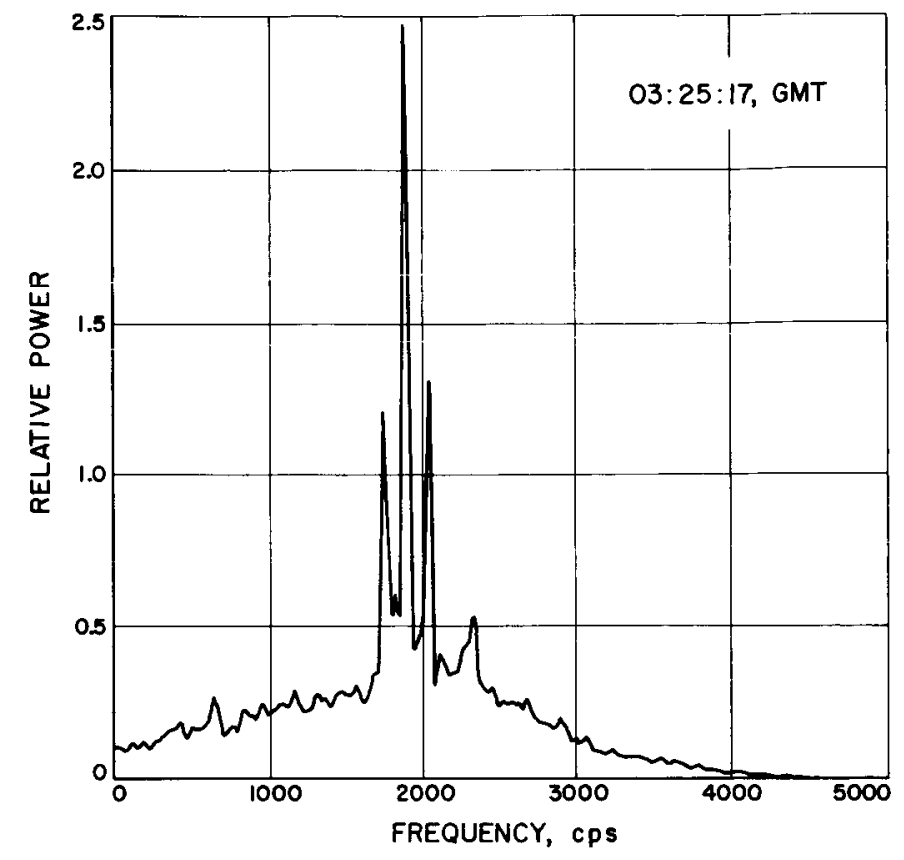

Fig. 24. Wide-band open-loop spectrum 3:25:17, DSIF-12 site

After the filtering, a high-resolution spectral analysis was performed for each 1-sec increment. Figure 25 illustrates a narrow-band spectrum of the same signal as in Fig. 24. A sinusoidal least squares fit was also performed

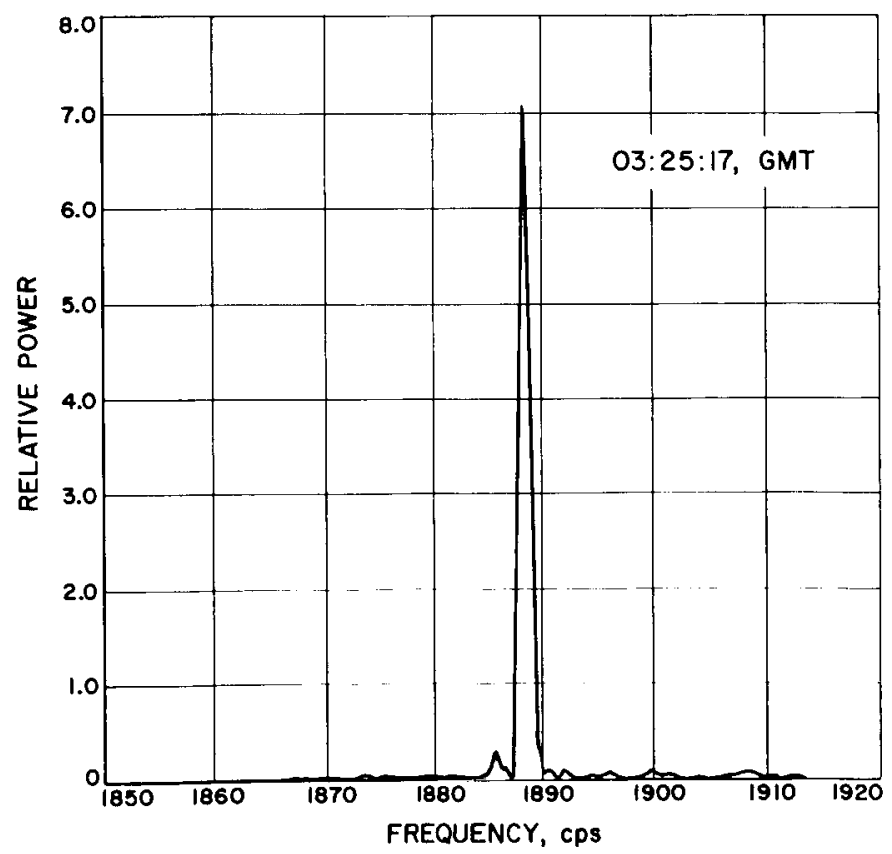

Fig. 25. Narrow-band open-loop spectrum 3:25:17, DSIF-12 site on each 1-sec increment. From these programs, the mean frequency as a function of time was obtained for both DSIF-11 and -12. The orbit-determination program was used to obtain doppler frequencies. From this, the predicted audio frequency was obtained and the residuals computed.

Figure 26 is a plot of the DSIF-12 residuals obtained on open- and closed-loop receivers. It can be seen that the agreement between the two techniques is excellent. It can also be seen that approximately one minute before occultation there was a perturbation, reportedly due to the Martian ionosphere.

After considerable experimentation, as described earlier, it was decided to attempt to illuminate Mars prior to the vehicle's emergence. It was hoped that the vehicle's radio would be in lock before the signal level of the received signal was above threshold. To help accomplish this, the $100-\mathrm{kw}$ transmitter of DSIF-13 was employed. On exit from occultation, the closed-loop receivers were not able to lock up rapidly enough to obtain tropospheric data. The open-loop receivers, however, were able to record it for post-processing.

Analysis of the data indicated that approximately $9 \mathrm{sec}$ of data were obtained with the crystal auxiliary oscillator driving the spacecraft transmitter before the spacecraft transponder went into lock. Figure 27 shows a 1-sec spectral analysis during which the crystal oscillator signal $(2.9 \mathrm{kc})$ stopped and the coherent signal $(1.9 \mathrm{kc})$ started. The modulation sidebands can be seen on both signals.

The auxiliary-oscillator signal is less desirable for two reasons. First, because there is only one path through the medium, the doppler effect is only one half as large as the coherent case. Second, we must assume that the crystal oscillator remained stable during the 9-sec interval.

The data received during emergence have been compiled. The one-way data have had a bias correction and the residuals have been corrected to the magnitude to be expected for two-way propagation. Figure 28 shows the residuals of DSIF-11 and -12 on emergence. The agreement between stations is excellent. It can be seen that the effects which have been interpreted as due to the Martian ionosphere are below threshold. The slope of the residuals through the troposphere are also somewhat different. Both of these results are possibly attributable to the fact that entrance to occultation took place in the daytime at the winter hemisphere and emergence occurred at night at the summer hemisphere. 


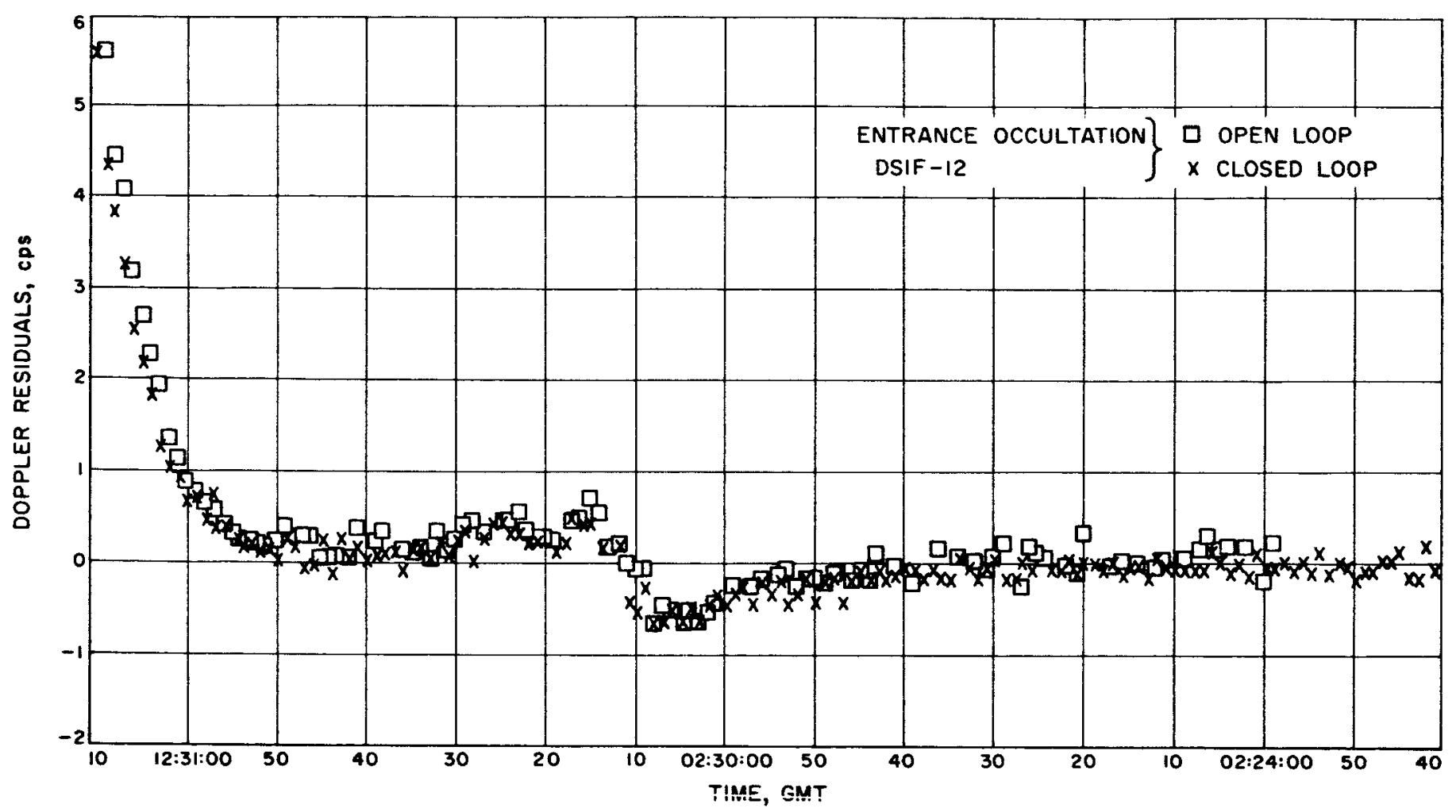

Fig. 26. Entrance occultation doppler residuals, DSIF-12 open- and closed-loop receivers

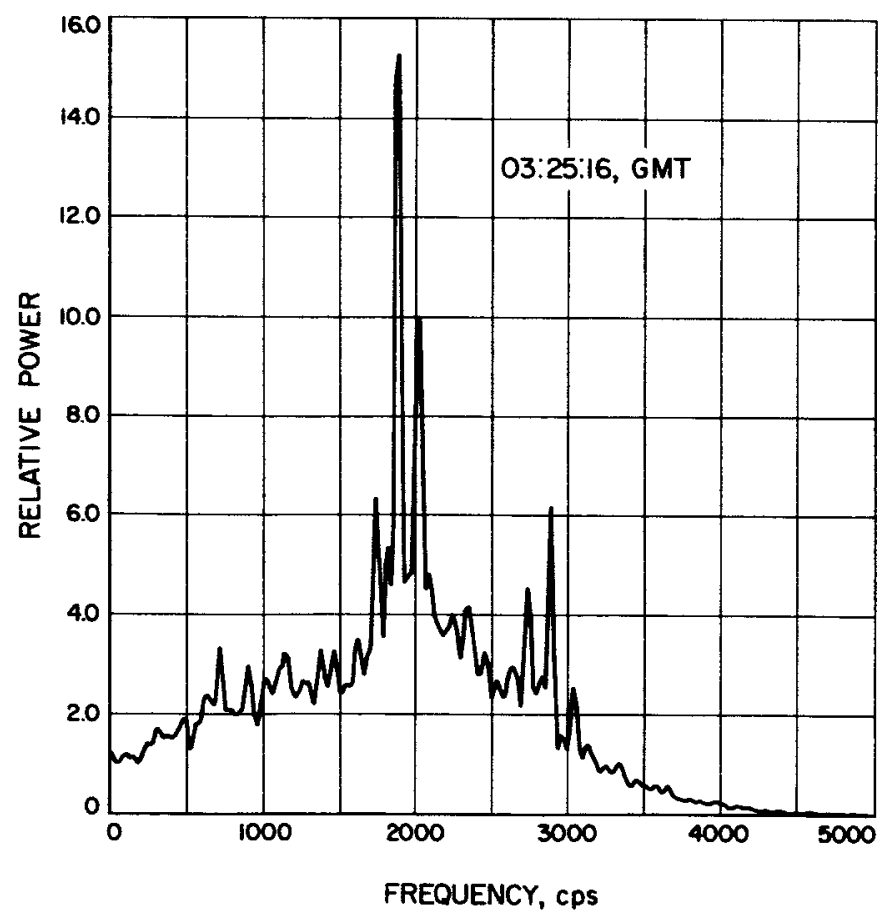

Fig. 27. Broad-band spectrum of transition from auxiliary oscillator to VCO 


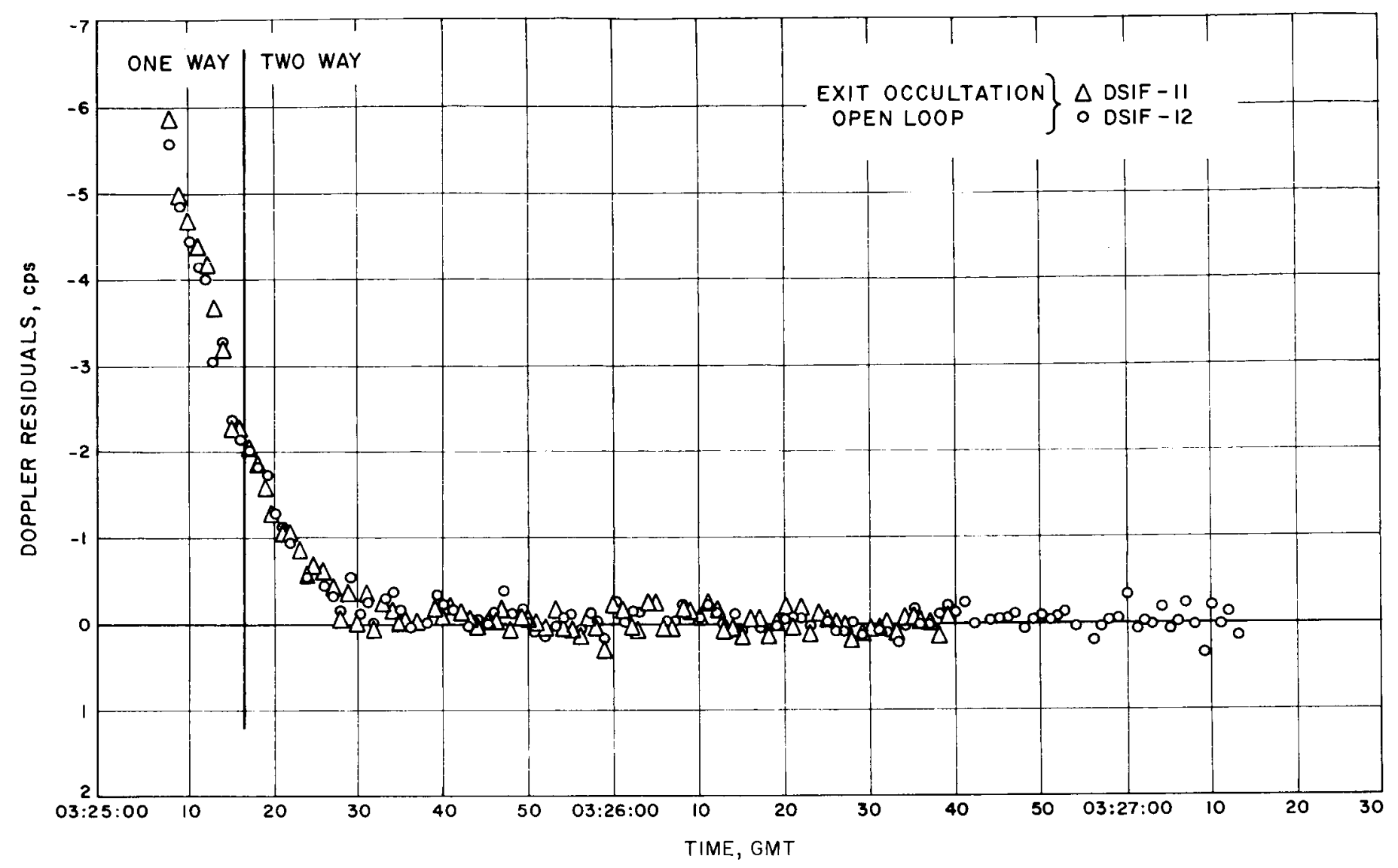

Fig. 28. Doppler residuals from exit of occultation from DSIF-11 and -12 open-loop receivers

\section{REFERENCES}

1. Kliore, A J., Cain, D. L., Levy, G. S., Eshleman, V. R., Drake, F. D., Fjeldbo, G., "The Mariner IV Occultation Experiment," Astronautics and Aeronautics, July 1965, pp. 72-80.

2. Kliore, A. J., Cain, D. L., Levy, G. S., Eshleman, V. R., Fieldbo, G., Drake, F. D., "Occultation Experiment: Results of First Direct Measurement of Mars' Atmosphere and lonosphere," Science, Vol. 149, 1965, pp. 1243-1248.

3. Fjeldbo, G., and Eshleman, V. R., The Bistatic Radar Occultation Method. A Study of Planetary Atmospheres, Scientific Report 5, Radio Science Laboratory, Stanford University, February 1965.

4. Victor, W. K., and Brockman, M. H., "The Application of Linear Servo Theory to the Design of AGC Loops," Proceedings of the IRE, Vol. 48, February 1960, pp. 234-238.

5. Viterbi, A. J., Acquisition Range and Tracking Behavior of Phase-Locked Loops, External Publication 673, Jet Propulsion Laboratory, Pasadena, California. 


\section{REFERENCES (Cont'd)}

6. Cain, D. L., Kliore, A. J., Levy, G. S., The Mariner IV Occultation Experiment: Summary of Data and Reduction Methods, AIAA Third Aerospace Sciences Meeting, January 24-26, 1966, Paper 66-148.

7. Fjeldbo, G., Fieldbo, W. C., Eshleman, V. R., "Models for the Atmosphere of Mars based on the Mariner IV Occultation Experiment," Journal of Geophysical Research, Vol. 71, May 1966, pp. 2307-2316.

\section{ACKNOWLEDGMENT}

The authors wish to express their appreciation for the assistance given by several people at Jet Propulsion Laboratory in this experiment: Robertson Stevens, M. Easterling, and Richard Goldstein for technical guidance; and D. Nixon for his aid in developing and testing the receiving equipment. They also wish to thank W. Silsby, J. Fulton, and the Data Analysis Laboratory.

The excellent cooperation of the Deep Space Network, particularly the personnel of the Pioneer and Echo sites, made this experiment possible. 